\title{
Investigation of Axial and Angular Sampling in Multi-Detector Pinhole-SPECT Brain Imaging
}

\author{
Navid Zeraatkar, Member, IEEE, Kesava S. Kalluri, Benjamin Auer, Arda Könik, Timothy J. Fromme, Lars R. \\ Furenlid, Phillip H. Kuo, and Michael A. King, Fellow, IEEE
}

\begin{abstract}
We designed a dedicated multi-detector multipinhole brain SPECT scanner to generate images of higher quality compared to general-purpose systems. The system, AdaptiSPECT-C, is intended to adapt its sensitivity-resolution trade-off by varying its aperture configurations allowing both high-sensitivity dynamic and high-spatial-resolution static imaging. The current system design consists of 23 detector heads arranged in a truncated spherical geometry. In this work, we investigated the axial and angular sampling capability of the current stationary system design. Two data acquisition schemes using limited rotation of the gantry and two others using axial translation of the imaging bed were also evaluated concerning their impact on image quality through improved sampling. Increasing both angular and axial sampling in the current prototype system resulted in quantitative improvements in image quality metrics and qualitative appearance of the images as determined in studies with specifically selected phantoms. Visual improvements for the brain phantoms with clinical distributions were less pronounced but presented quantitative improvements in the fidelity (normalized root-mean-square error (NRMSE)) and striatal specific binding ratio (SBR) for a dopamine transporter (DAT) distribution, and in NRMSE and activity recovery for a brain perfusion distribution. More pronounced improvements with increased sampling were seen in contrast recovery coefficient, bias, and coefficient of variation for a lesion in the brain perfusion distribution. The negligible impact of the most cranial ring of detectors on axial sampling, but its significant impact on sensitivity and angular sampling in the cranial portion of the imaging volume-of-interest were also determined.
\end{abstract}

Index Terms-AdaptiSPECT-C, angular and axial sampling, SPECT, pinhole, multi-pinhole.

\section{INTRODUCTION}

B RAIN SPECT imaging has been of interest for many clinical applications in neuroimaging including Alzheimer's, Parkinson's, and Huntington's diseases,

Research reported in this publication was supported by the National Institute of Biomedical Imaging and Bioengineering of the National Institutes of Health under Award Number R01 EB022521. The content is solely the responsibility of the authors and does not necessarily represent the official views of the National Institutes of Health.

N. Zeraatkar, K. S. Kalluri, B. Auer, and M. A. King are with the Department of Radiology, University of Massachusetts Medical School, Worcester, MA 01605 USA (e-mail: Navid.Zeraatkar@umassmed.edu).

T. J. Fromme is with the Worcester Polytechnic Institute, Worcester, MA 01609 USA.

A. Könik is with the Department of Imaging, Dana Farber Cancer Institute, Boston, MA 02215 USA.

L. R. Furenlid is with James C. Wyant College of Optical Sciences and the Department of Medical Imaging, University of Arizona, Tucson, AZ 85724 USA.

P. H. Kuo is with the Department of Medical Imaging, University of Arizona, Tucson, AZ 85724 USA.

Manuscript received Apr 04, 2020; revised Jul 23, 2020. attention-deficit/hyperactivity disorder (ADHD), and tumor imaging [1-7]. In addition, ictal versus interictal SPECT imaging with brain perfusion agents plays an important role in localizing the region of seizure onset $[8,9]$.

There has been a trend toward the development of dedicated brain SPECT scanners with higher sensitivity and superior resolution [10], instead of using general-purpose SPECT scanners for brain imaging. Among the earliest systems, the Mark IV system may be considered one of the pioneers in this category, capable of imaging the brain using radionuclide imaging agents available at that time [11]. Neurocam (GE-CGR, Buc, France) [12], a stationary hemispherical SPECT imager built at the University of Arizona [13], and CERASPECT [14] were other examples of dedicated brain SPECT scanners with fully stationary or pseudo-stationary gantries. More recently, other systems have been developed as well [10, 15-17]. From the early days of SPECT significant work has also been performed on perfecting the collimation employed with existing multiheaded rotating SPECT systems for usage in brain imaging. For example, slant-hole collimators were investigated to allow the detector heads to clear the shoulders of patients and yet have the collimator close to the superior portion of the head when rotating around patients $[18,19]$. Cone-beam and fanbeam collimators were used to make better usage of the detector area than parallel-hole collimators when imaging a structure such as the head which is of smaller area than the detector, thereby improving the sensitivity / spatial resolution trade-off for imaging [20, 21]. More recently, studies have shown that customized multi-pinhole collimators may further enhance the performance of the existing clinical generalpurpose SPECT systems for brain imaging [22-26].

We are designing a stationary brain-dedicated SPECT system, AdaptiSPECT-C, with high resolution and sensitivity to allow static and dynamic imaging [27, 28]. The scanner is being designed to use multiple detectors with multiple pinhole apertures per detector. The aperture configurations (i.e. sizes and acceptance angles) can be varied, and opened or closed through a shuttering mechanism [29]. The current prototype design for AdaptiSPECT-C is described in section II.A.

In computed tomography (CT) with a cone-beam source which emits photons as a three-dimensional cone, it has been shown that data sampling completeness can only be achieved in the plane in which the cone-beam sources are located [30]. By analogy, in pinhole SPECT one can envision the aperture as transmitting to the detector information about the source in front of the aperture and that data sampling would only be complete in the transverse plane of the ring of 

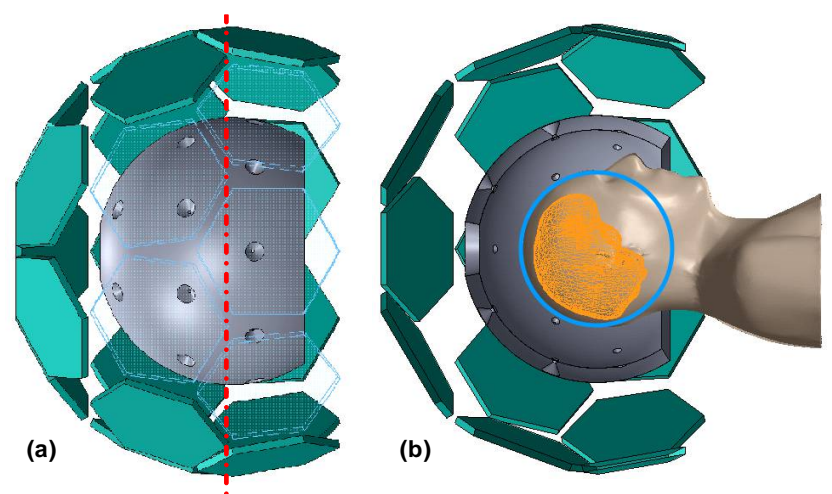

Fig. 1. (a) Current prototype design of the AdpatiSPECT-C system with 23 hexagonal detectors in three rings, orthogonal to the imaging bed axis, named quasi-vertex $(\mathrm{QV})$, middle, and caudal respectively from left to right in the figure. The equatorial plane is shown by the dashed dotted line. Note five detectors are made invisible to enable visualization of the collimator and apertures. (b) Typical positioning of patient inside the system is shown by a virtual cut-plane. The blue circle shows the sagittal cut-plane through the center of the $21-\mathrm{cm}$ dimeter spherical volume-of-interest (VOI) encompassing the brain (shown in orange) of the $50^{\text {th }}$ percentile size in men [32] for SPECT imaging. Note although the $50^{\text {th }}$ percentile size in men is used in simulations in this study, the $21-\mathrm{cm}$ diameter spherical VOI of the AdaptiSPECT-C is large enough to image the brain of the $99^{\text {th }}$ percentile size in men [32] without truncation.

apertures. Angular sampling conditions are also important for cone-beam-based collimators. The required number of angular samples depends on the object's size, shape, and activity distribution, the imaging task, and available prior information [31].

In this work, we investigate axial and angular sampling for the AdaptiSPECT-C current system prototype, based on the reconstructed image quality. We also explore how enhancing the sampling can affect the image quality. For this we examine the impact of two approaches based on rotation of gantry and axial translation of imaging bed on angular and axial sampling, respectively.

\section{Methods}

\section{A. AdaptiSPECT-C Brain SPECT Imaging System}

We have evaluated several configurations for AdaptiSPECTC. The current prototype design, as illustrated in Fig. 1(a), is a truncated spherical geometry with 23 "detector heads" (detector and corresponding pinhole aperture) in a stationary gantry. In this design, the detector heads are arranged in three rings orthogonal to the imaging bed axis. We refer to these rings as the caudal, middle, and quasi-vertex (QV) rings, respectively, by their location along caudo-cranial direction. While the caudal and middle rings consist of 9 detector heads, each $40^{\circ}$ azimuthal from adjacent ones, the QV ring has only 5 detector heads, each $72^{\circ}$ azimuthal from adjacent ones. The detector heads in the caudal ring have a $20^{\circ}$ azimuthal difference relative to the ones in the middle ring. Assuming the equatorial plane is set at $0.0 \mathrm{~cm}$ and positions cranial to this plane being positive, the axial positions of the detector centers in the caudal, middle, and QV rings are -5.0, 9.8, and $24.4 \mathrm{~cm}$, respectively. Similarly, the axial positions of the apertures in the caudal, middle, and QV rings are -3.1, 6.2, and $15.3 \mathrm{~cm}$, respectively. Although AdaptiSPECT-C is being designed with multiple configurable pinholes per detector, in the current prototype design investigated herein each detector head consists of a hexagonal detector $(20 \mathrm{~cm}$ vertex-to-vertex $)$ and a single direct pinhole aperture (double knife-edge, $4 \mathrm{~mm}$ in diameter with $75^{\circ}$ opening angle). All apertures are focused at the center of a 21 -cm-diameter spherical volume-of-interest (VOI). Aperture-center-to-VOI-center and detector-surface-toVOI-center distances are 18.0 and $28.7 \mathrm{~cm}$, respectively. With these parameters, even the brain of the $99^{\text {th }}$ percentile size in men [32] fits within the VOI and can be images by all the 23 detector heads without truncation. Fig. 1(b) shows patient $\left(50^{\text {th }}\right.$ percentile size in men [32] which is used in this study) placement and the spherical VOI.

\section{B. Digital Phantoms}

We used a set of voxelized phantoms for evaluating the effect of different sampling schemes on the reconstructed images. The phantoms we used were a sphere of uniform activity, a hot-rod, Defrise, and three anthropomorphic brain phantoms as described below.

To analyze the impact of the number of angular samples, a phantom of uniform activity was used. Insufficient sampling then presents in the reconstructed images as non-uniformity artefacts [31]. We used a sphere phantom (radius: $10 \mathrm{~cm}$ ) of uniform ${ }^{123} \mathrm{I}$ activity (simulated $159 \mathrm{keV}$ emissions and attenuation only) in water for this purpose.

A phantom composed of an array of equidistant and equaldiameter hot-rods (hot-rod phantom) was also designed to investigate the impact of angular sampling on image quality within the imaging VOI. As shown in Fig. 2(a), a total of 121 hot rods, $8 \mathrm{~mm}$ in diameter and $195 \mathrm{~mm}$ in length, were fit in a cylindrical container. The center-to-center distance between adjacent rods is 2 times the diameter of the rods. The outer diameter and height of the container were both set to $201 \mathrm{~mm}$. The rods were simulated as filled by uniform activity of ${ }^{123} \mathrm{I}$ in water while the rest of the phantom had no activity. An attenuation coefficient equivalent to that of water for ${ }^{123} \mathrm{I}(\mu=$ $0.147 \mathrm{~cm}^{-1}$ for $159 \mathrm{keV}$ ) was employed for the cylindrical container in simulation and reconstruction.

To assess the impact of variations in axial sampling, we generated a customized cylindrical Defrise phantom [21]. The digital Defrise phantom employed herein is based on Defrise Insert ${ }^{\mathrm{TM}}$ Model ECT/DEF/I (Data Spectrum Corporation, Durham, NC, USA). The original Defrise Insert ${ }^{\mathrm{TM}}$ consists of 9 solid disks, and hence 8 gaps which are filled by activity in water. We will refer to these activity-filled gaps as "hot disks" in this manuscript. To customize it for the 210-mm axial extent of the AdaptiSPECT-C imaging VOI, we added one more solid disk to each side of the phantom resulting in total number of 11 solid disks and 10 hot disks. The diameter and thickness of the solid disks were 209 and $12 \mathrm{~mm}$, respectively. The centerto-center spacing between the adjacent solid disks was set to $20 \mathrm{~mm}$ forming hot disks of $8 \mathrm{~mm}$ thickness. We modeled the Defrise phantom inside a cylindrical container with inner diameter and wall thickness of 216 and $3 \mathrm{~mm}$, respectively. The phantom was simulated to be filled by uniform activity 


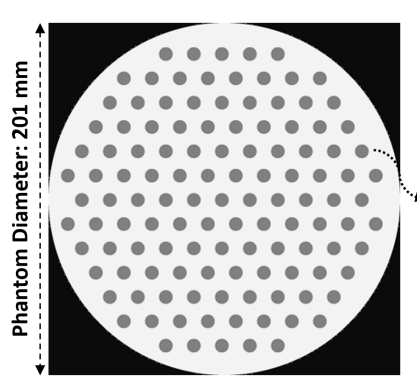

(a)

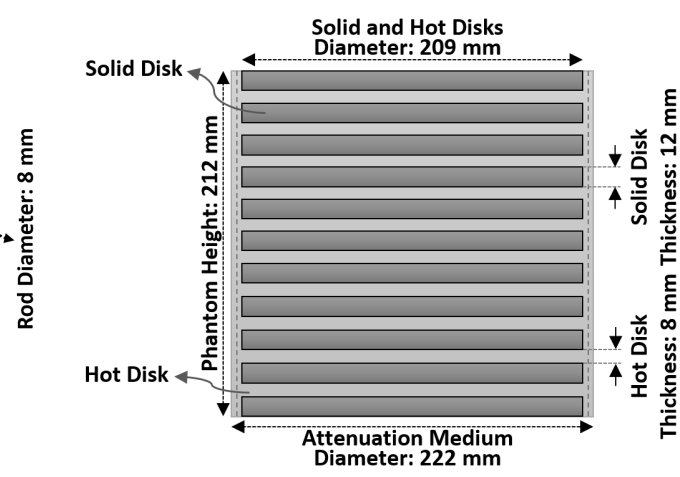

(b)

Fig. 2. (a) Transverse view of the hot-rod activity phantom designed for evaluation of angular sampling. (b) Mid-coronal view of the Defrise phantom with 11 solid and 10 hot disks customized based on Defrise Insert ${ }^{\mathrm{TM}}$ Model ECT/DEF/I (Data Spectrum Corporation, Durham, NC, USA) to evaluate axial sampling. Notice that herein we refer to the activity-filled gaps between the solid disks as "hot disks."

of ${ }^{123} \mathrm{I}$ in water which created the hot disks. An attenuation coefficient equivalent to that of water for ${ }^{123}$ I was employed for all attenuation media in the phantom (including solid disks and cylindrical container) in simulation and reconstruction. Fig. 2(b) shows a drawing of the customized Defrise phantom.

To inspect the impact of axial and angular sampling on brain SPECT images, three brain phantoms were generated using the extended cardiac-torso (XCAT) digital phantom [33]: first brain phantom models the activity distribution of [I-123] Nisopropyl iodoamphetamine (I-123 IMP) for brain perfusion imaging with a "gray-matter:white-matter:head-soft-tissue" activity concentration ratio of 4:1:0.4 [34]. Second brain phantom models a lesion in the brain perfusion phantom to investigate the effect of axial and angular sampling on imaging an abnormality. The lesion was modeled as a hot sphere, $3.2 \mathrm{~cm}$ in diameter. Since temporal lobe epilepsy is the most common form of epilepsies [35], in this work we placed the lesion in the temporal lobe. The "lesion:gray-matter:white-matter:headsoft-tissue" activity concentration ratio was 8:4:1:0.4. Third brain phantom models the activity distribution for [I-123]ioflupane dopamine active transporter (DAT) imaging with a "striatum:background:head-soft-tissue" activity concentration ratio of 8:1:0.5 [36]. The background for DAT phantom stands for the non-striatal part of the brain. The same attenuation map was considered for the brain perfusion (with and without the lesion) and DAT phantoms. The brain phantom sizes were based on the head sizes of $50^{\text {th }}$ percentile in men [32] and were positioned so that the cranial region was aligned to the superior axial extent of the imaging VOI, mimicking clinical imaging, as illustrated in Fig. 1(b).

All voxelized activity and attenuation phantoms described in this section were modeled with voxel size of $(0.5 \mathrm{~mm})^{3}$.

\section{Definition of Quantitative Metrics}

The first metric we used to compare sampling schemes is the normalized root-mean-square error (NRMSE). The NRMSE was calculated as an index of the fidelity with which the source distribution with the same voxel dimensions as the reconstruction was estimated in the reconstructed slices. It was defined as:

$$
N R M S E=\sqrt{\frac{\sum_{n=1}^{N}\left(I(n)-I_{G T}(n)\right)^{2}}{\sum_{n=1}^{N}\left(I_{G T}(n)\right)^{2}}}
$$

where $I, I_{G T}, n$, and $N$ are the reconstructed image, the ground truth image, the voxel index, and total number of voxels in the image volume, respectively.

We also calculated bias and coefficient of variation $(\mathrm{CV})$ as measures of accuracy and fluctuations in reconstruction, respectively. Bias was computed as:

$$
\operatorname{Bias}(\%)=\frac{a b s\left(\text { mean }_{I}-\text { mean }_{I_{G T}}\right)}{\text { mean }_{I_{G T}}} \times 100
$$

where mean and $_{I}$ mean ${ }_{I_{G T}}$ denote the mean value of the reconstructed image and the ground truth image, respectively, over the volume of calculation.

$\mathrm{CV}$ was calculated as:

$$
C V(\%)=(\sigma / \mu) \times 100
$$

in which $\sigma$ and $\mu$ are standard deviation and mean, respectively, over the volume of calculation.

Non-uniformity was also calculated for the images of the sphere phantom as:

$$
\text { Non-Uniformity }(\%)=\frac{\max -\min }{\max +\min } \times 100
$$

where maximum and minimum were calculated over the volume of calculation.

Contrast recovery coefficient (CRC), used for quantitative analysis of the lesion detectability in the brain perfusion phantom, was defined as:

$$
C R C(\%)=\frac{\left(\text { mean }_{H o t} / \text { mean }_{B k g}\right)-1}{\left(A_{H o t} / A_{B k g}\right)-1} \times 100
$$

where mean $_{H o t}$ and mean $_{B k g}$ are the mean value of hot and background VOI, respectively, and $A_{H o t} / A_{B k g}$ is the ratio of the true hot and background activity concentrations.

Brain perfusion imaging can be employed for dementia differentiation, cerebrovascular disease evaluation, epileptogenic 
foci localization, diagnosis of movement disorders, and tumor evaluation [37]. To quantify accuracy in recovering the activity concentration, we defined Activity Recovery $(\%), \operatorname{AR}(\%)$, as the percentage of reconstructed activity to true activity within a given anatomical brain region. We defined 18 3D regions as shown in Fig. 3, based on the anatomical volumes occupied in XCAT phantom. The AR\%, with the true value of $100 \%$, can show the accuracy of a sampling scheme in recovering the activity in a brain region.

Semiquantitative analysis of DAT images using striatal specific binding ratio (SBR) has been shown to be helpful for reporting of clinical cases [38, 39]. We calculated SBR as:

$$
S B R=\frac{\text { mean }_{\text {striatal-activity }}-\text { mean }_{\text {occipital-activity }}}{\text { mean }_{\text {occipital-activity }}}
$$

where striatal activity included caudate and putamen activities. Occipital lobe and striatal activities were calculated using the 3D definitions of the regions as shown in Fig. 3 and described above.

\section{Simulation and Image Reconstruction}

An in-house analytical simulation and iterative reconstruction software package developed for pinhole collimators and validated against Monte Carlo (MC) simulations and MCbased system matrix reconstruction, respectively, [40] was employed in our investigations. The software estimates the system matrix for simulation and reconstruction on-the-fly based on multi-ray-tracing. It was implemented exploiting the parallel computation power of graphics-processing-units (GPU) to achieve fast simulation and reconstruction. The physical and geometric properties of the system were modeled in the simulator and reconstruction software, including system geometry and dimensions, detector size, intrinsic blurring and detection efficiency, and aperture size and opening angle for knife-edge apertures. Object attenuation was also modeled in the simulations and corrected for in the reconstructions based on a fast single-ray-tracing approach [41]. Since the main motivation of this work was to assess the sampling issues of the system, scatter (within system and phantom) and energy resolution were not included in simulations. Poisson noise was modeled in simulations of the brain perfusion and DAT phantoms as explained in detail in section II.E. A projection matrix of $200 \times 200$ with a pixel size of $(1.0 \mathrm{~mm})^{2}$ was used for acquisition by each detector head.

The image reconstruction was based on maximumlikelihood expectation-maximization (MLEM) algorithm [42, 43] where the forward- and back-projection operators were implemented in the same way as the analytic simulator. The voxel size of the phantoms in the simulations and the reconstructions were $(0.5 \mathrm{~mm})^{3}$ and $(1.0 \mathrm{~mm})^{3}$, respectively, satisfying Nyquist-Shannon sampling theorem [44] and approximating a continuous to discrete transformation in imaging [45]. No pre- or post-reconstruction filter was applied.

In this work, we simulated several data acquisition schemes. Therefore, the reconstruction convergence rate for each case was different. All the reconstructions herein were allowed 150 iterations. We defined iteration-of-interest as the iteration at
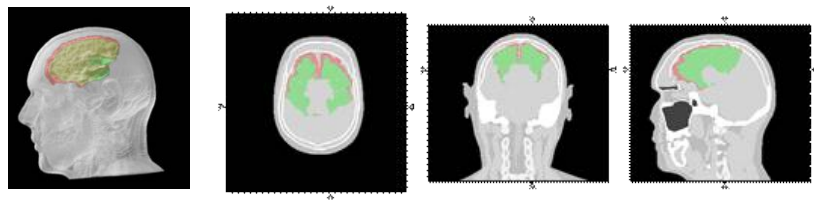

Frontal lobe white and gray matter
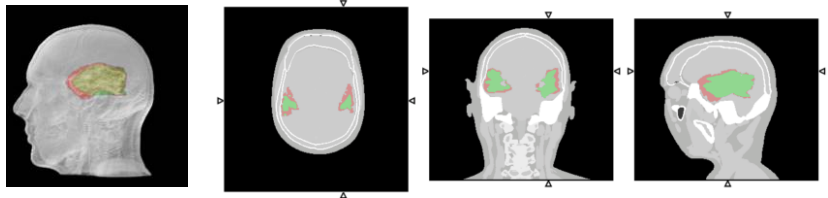

Temporal lobe white and gray matter
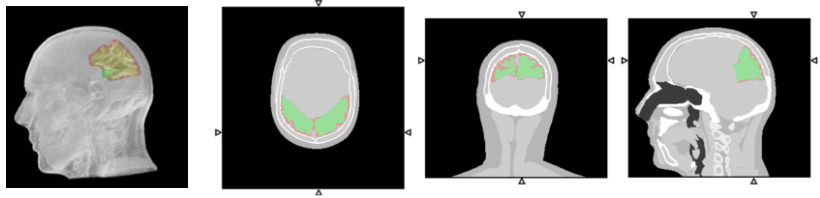

Parietal lobe white and gray matter
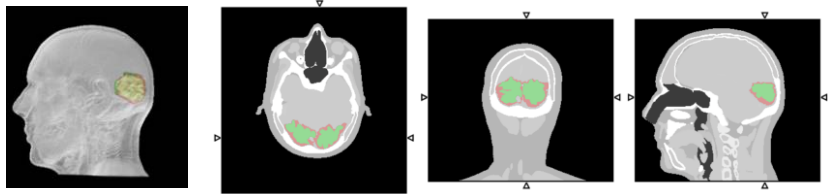

Occipital lobe white and gray matter
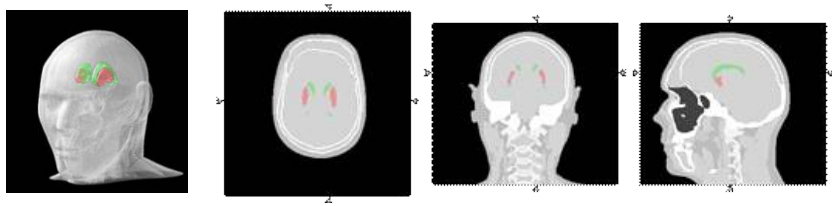

Caudate and putamen
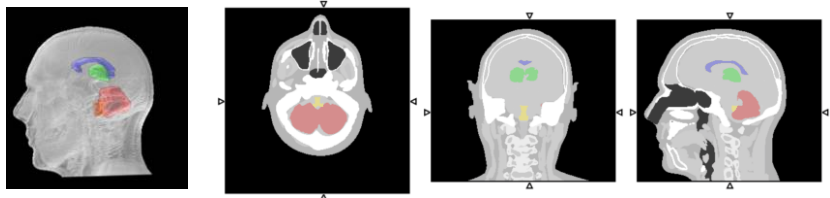

Cerebellum, corpus callosum, thalamus, and medulla oblongata
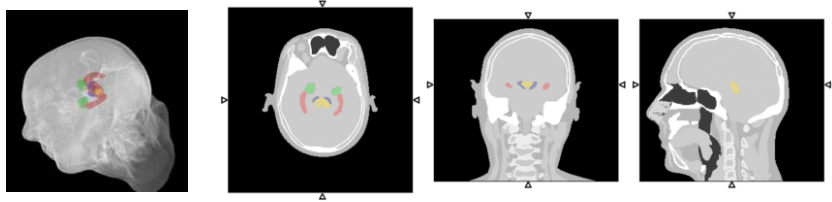

Hippocampus, substantia migra, Amygdala, and midbrain

Fig. 3. The 18 three-dimensional (3D) brain regions derived from the XCAT brain are shown overlaid on the attenuation map of the head. The first column shows 3D-rendering of the regions while the $2^{\text {nd }}$ to $4^{\text {th }}$ columns show sample transverse, coronal, and sagittal slices, respectively. In the brain lobes, white and gray matters are shown in green and red, respectively. The yellow regions seen in the brain lobes represent regions of white and gray matters overlap in display.

which we performed both qualitative and quantitative analyses of the reconstructed images as: the iteration with the minimum NRMSE (point of inflection) if the NRMSE versus iterations plot was convex. However, if the plot was asymptotic over the 150 iterations, the iteration-of-interest was defined as the earliest iteration where the mean change in voxel values of the image [46] was less than $1 \%$ as compared to the previous iteration. We calculated the mean change as below: 


$$
(\text { Mean Change })_{i t r}=\operatorname{mean}\left(\sum_{n=1}^{N} a b s\left(I_{i t r}(n)-I_{i t r-1}(n)\right)\right)
$$

where itr is the iteration number varied from 2 to the total number of iterations (i.e., 150). For the mean change calculation, we excluded the image voxels that lie outside the radius of $11.5 \mathrm{~cm}$ from the focal spot. This was achieved by gradually rolling off the post-reconstruction values of image voxels between radius of 10.5 and $11.5 \mathrm{~cm}$, and setting to zero the values of the voxels beyond $11.5 \mathrm{~cm}$. We will refer to this procedure as image trimming in the remainder of the manuscript.

\section{E. Sampling in the Current Design of the AdaptiSPECT-C}

As mentioned in section II.A and illustrated in Fig. 1, our current prototype AdaptiSPECT-C system design is based on the arrangement of the detector heads in a truncated spherical structure around the spherical VOI with the central axes of all pinholes intersect at the center of this VOI. In this section, the sampling capability of the current prototype system with a single aperture per detector is investigated. Also, to analyze the impact of enhanced axial and angular sampling on the reconstructed images, two approaches are investigated in terms of their effect on image quality.

If the current configuration of the AdaptiSPECT-C is angularly under-sampling, acquiring additional angular views by rotation of the system around the axial direction would thus enable enhancing the angular sampling leading to an improvement in image quality. Similarly, increasing the number of axial samples using axial translation either of the system or the object to extra axial positions, would improve image quality if the original design had substantial axial undersampling. These assumptions regarding the impact of limited rotation and translation on image quality were evaluated as follows. For rotating the system around the caudo-cranial axis we simulated: $i$ ) two angular positions with a $10^{\circ}$ azimuthal step, and ii) four angular positions with three $5^{\circ}$ azimuthal steps. Please recall that there is a $20^{\circ}$ azimuthal angulation between the detector heads of the caudal and middle rings. Thus, these two schemes result in the caudal and middle rings' projections being acquired at $10^{\circ}$ and $5^{\circ}$ azimuthal angulation steps, respectively. For the axial translation, we modeled: $i$ ) two axial positions, one with the object at the axial center, and the other with $4.6 \mathrm{~cm}$ axial translation, inward (i.e., toward the QV ring), and ii) three axial positions, one with the object at the axial center and two others with axial translations of respectively 3.1 and $6.2 \mathrm{~cm}$ inward. Since the axial difference between the QV-to-middle and middle-to-caudal apertures is about 9.1 and $9.3 \mathrm{~cm}$, respectively, by setting the step size to $4.6 \mathrm{~cm}$ (i.e., $9.2 \mathrm{~cm} \mathrm{/2)} \mathrm{for} \mathrm{the} \mathrm{first} \mathrm{axial} \mathrm{translation} \mathrm{scheme}$ and $3.1 \mathrm{~cm}$ (i.e., $9.3 \mathrm{~cm} / 3$ ) for the second scheme, we tried to acquire evenly distributed axial samples over the imaging VOI. For simulation of the schemes with more than one step (translation/rotation), the same activity level and total scan time as the stationary scan (no rotation/translation) was considered. The total scan time was equally divided between each translation/rotation step. The overhead time taken for gantry/object rotation/translation was assumed to be negligible for these simulation studies.

Since more scan steps result in less acquired counts per step, in realistic scans the increased noise in each projection might compromise the potential improvement from increased sampling. To investigate this, we modeled 5 Poisson noise realizations at realistic count levels for the brain perfusion and DAT simulations. According to guidelines, for DAT and perfusion SPECT imaging a minimum of 1.5 and 5 million (M) counts, respectively, should be acquired [47, 48]. We simulated $3 \mathrm{M}$ and $10 \mathrm{M}$ total counts for DAT and brain perfusion imaging, respectively, for the stationary data acquisition. These count levels satisfy the required minimum counts and are in accordance with the typical count levels acquired in our department. Please note the total acquired counts for the simulated acquisition schemes for axial sampling evaluation (i.e., 2 and 3 axial positions) are different regarding their sensitivity relative to the stationary acquisition due to truncation of the VOI with axial motion. Volumetric sensitivity for these three acquisition schemes was defined as the total sensitivity over the 21cm-diameter spherical imaging VOI. This was calculated by summing the sensitivity values of all voxels in the imaging VOI incorporating uniform attenuation $\left(\mu=0.147 \mathrm{~cm}^{-1}\right)$ within this VOI.

For the sphere, hot-rod, Defrise, and brain perfusion phantoms, the NRMSE was calculated for the reconstructed images over a 22-cm diameter sphere (i.e., slightly larger than the 21$\mathrm{cm}$ diameter spherical imaging VOI of the AdaptiSPECT-C). For the DAT phantom, the NRMSE was calculated over a volume including $i$ ) an 8 -cm diameter sphere encompassing the striatal region and $\mathrm{ii}$ ) subcortical occipital lobes of the brain. The rationale for choosing such a volume for NRMSE calculation in DAT phantom is the importance of those regions for SBR calculation. For the sphere phantom, to avoid the effects of Gibbs artefact in the reconstructed images at the phantom boundaries, an $18-\mathrm{cm}$ diameter spherical volume of calculation was used for the bias, $\mathrm{CV}$, and non-uniformity.

For the reconstructed images of the brain perfusion phantom with lesion, the $\mathrm{CRC}(\%)$ was calculated using Eq. 5 by defining the hot VOI as a sphere concentric to the lesion, 0.9 times the size of the original spherical lesion. The background VOI was defined as a sphere of the same size as the hot VOI over the cerebellum. The hot and background VOI for calculating $\mathrm{CRC}(\%)$ are shown in Fig. 9(a). The bias and CV for the lesion were also calculated over the hot spherical VOI.

\section{F. Contribution of the Quasi-Vertex (QV) Ring of the Detector Heads in Sampling}

The motivation to have the QV ring of the detector heads in the AdaptiSPECT-C is to improve sensitivity for brain imaging [28], particularly for cranial regions. However, since the axial position of the apertures in the $\mathrm{QV}$ ring (i.e., $15.3 \mathrm{~cm}$ ) is beyond the axial extent of the imaging VOI (i.e., $10.5 \mathrm{~cm}$ ), the advantage of the QV ring in terms of axial sampling is not very clear. To investigate the contribution of the $\mathrm{QV}$ ring towards axial sampling, we simulated the Defrise phantom in 
the AdaptiSPECT-C with and without the 5 detector heads in the QV ring. Similarly, to evaluate the QV detector heads' contribution towards angular sampling, we simulated the hotrod phantom in the current design of the AdaptiSPECT-C with and without the $5 \mathrm{QV}$ detector heads. The simulation and reconstruction parameters were the same for both geometries with and without the $5 \mathrm{QV}$ detector heads. The volumetric sensitivity was also calculated, as described in section II.E, with and without the QV ring.

\section{RESULTS}

\section{A. Sampling in the Current Design of the AdaptiSPECT-C}

Fig. 4(a) shows the central transverse reconstructed slices of the sphere phantom at the iteration-of-interest for the stationary configuration (i.e., without rotation or translation), and with the two gantry rotation approaches. For the sphere phantom, the uniformity of the central transverse slice improves as the number of gantry angular positions increase from 1 to 4, especially with two gantry positions and to a lesser extent with four gantry positions. This agrees with the non-uniformity values plotted in Fig. 4(b). The slightly higher non-uniformity $(0.16 \%)$ observed with 4 angular positions compared to 2 angular positions may be related to the manner the VOI was defined (i.e., an $18-\mathrm{cm}$ diameter sphere) for non-uniformity calculation (see section II.E) excluding the uniformity enhancement in voxels lying outside a diameter of $18 \mathrm{~cm}$ in the reconstructed images of the sphere phantom. This follows the improvement in the NRMSE, bias, and CV values in bar graphs shown in Fig. 4 (c)-(e), respectively. The NRMSE values at the iteration-of-interest for stationary (i.e., one angular position), two angular positions, and four angular positions for the sphere phantom were 0.0138 at iteration 70, 0.0071 at iteration 25, and 0.0067 at iteration 24 , respectively. The bias for the phantom with one, two, and four angular positions at the iteration-of-interest, as reported earlier, were $0.485,0.230$, and 0.226 , respectively. The CV for one, two, and four angular positions were computed as $1.28 \%, 0.67 \%$, and $0.63 \%$, respectively, at the iteration-ofinterest. The non-uniformity for one, two, and four angular positions at the iterations-of-interest were $9.06 \%, 3.76 \%$, and $3.92 \%$, respectively.

Fig. 5(a) shows the central transverse reconstructed slices of the hot-rod phantom for the stationary system configuration, as well as for the two data acquisition schemes with gantry rotation. Only for display, the image trimming was applied on the reconstructed images. Note although the original hot-rod phantom used in the simulation is cylindrical (see section II.B), to generate the ground truth image, we down-sampled the activity phantom to match the dimensions of the reconstructed image and then applied image trimming. The corresponding ground truth slice is also shown in Fig. 5(a). We summed all the transverse slices and from the resultant slice, midhorizontal profile along the arrow shown on the ground truth image in Fig. 5(a), was plotted in Fig. 5(b). The enhancement in rod visibility across the slice with improving angular sampling is observed in the displayed slices, as well as in the profile on the axially summed transverse slice, especially

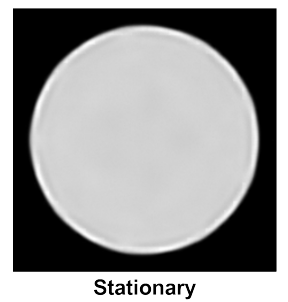

Stationary
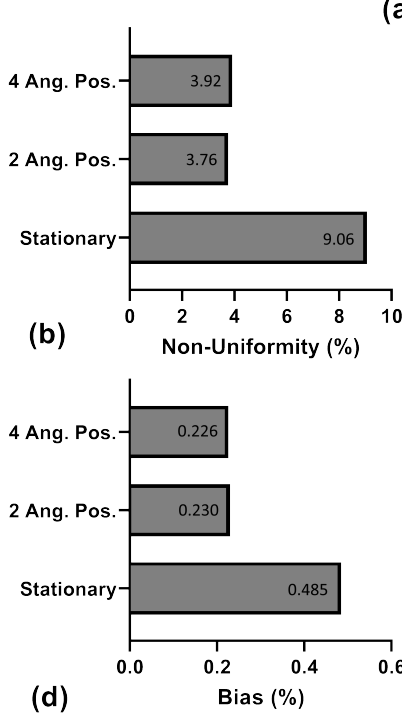

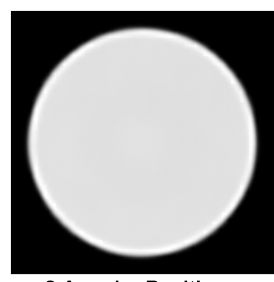

2 Angular Positions

(a)
4 Angular Positions
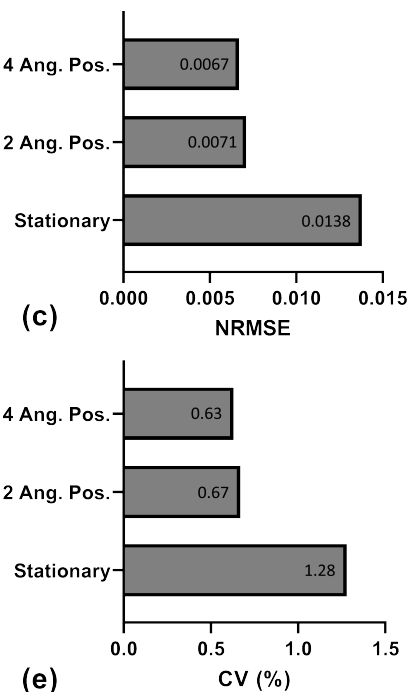

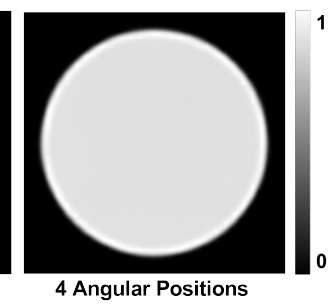

Fig. 4. (a) Central transverse slices of the $20-\mathrm{cm}$ diameter sphere phantom for the current prototype design of the AdaptiSPECT-C with a stationary scan (no rotation), using data acquisition scheme with two angular positions with $10^{\circ}$ azimuthal between, and four angular positions with $5^{\circ}$ azimuthal between. (b)-(e) The non-uniformity, NRMSE, bias, and CV, respectively, at the iteration-of-interest with different number of angular positions in data acquisition. Notice the substantial image improvement with adding an additional angular rotation, and modest additional benefit for three rotations.

in the central region of the phantom. The same trend is seen in the NRMSE values plotted in Fig. 5(c). The NRMSE values at the iteration-of-interest for the stationary, two angular positions, and four angular positions were calculated as 0.6923 at iteration $136,0.5558$ at iteration 135 , and 0.5251 at iteration 134 , respectively.

Fig. 6(a) shows the central coronal reconstructed slice of the Defrise phantom for the stationary system configuration, as well as for the two data acquisition schemes employing axial translation of the imaging bed. Only for display, the image trimming was applied on the reconstructed images. Although the original Defrise phantom used in the simulation is cylindrical (see section II.B and Fig. 2(b)), it was down-sampled to match the dimensions of the reconstructed image and then image trimming was applied (as described in section II.D) to generate the ground truth image. The corresponding slice of this ground truth image is shown in Fig. 6(a). We generated a summed axial profile of the reconstructed images and the corresponding one for the ground truth image by representing each transverse slice by the sum of its voxels. These summed axial profiles are shown in Fig. 6(b). Note that regarding the summed axial profile, only 4 disks were distinguished using stationary acquisition (i.e., one axial position) whereas with two and three axial positions, 9 disks (i.e., all disks except 
(a)

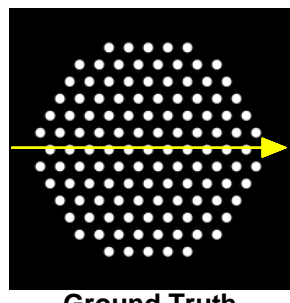

Ground Truth

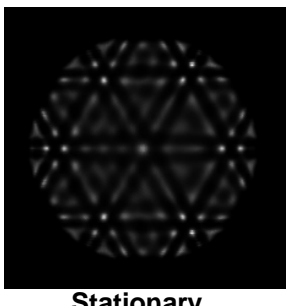

Stationary

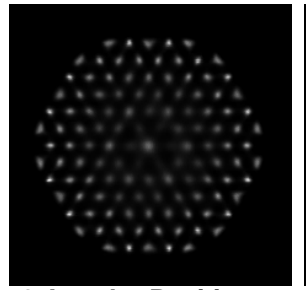

2 Angular Positions

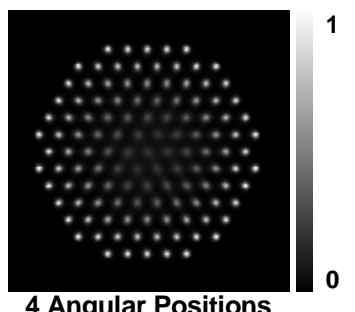

4 Angular Positions
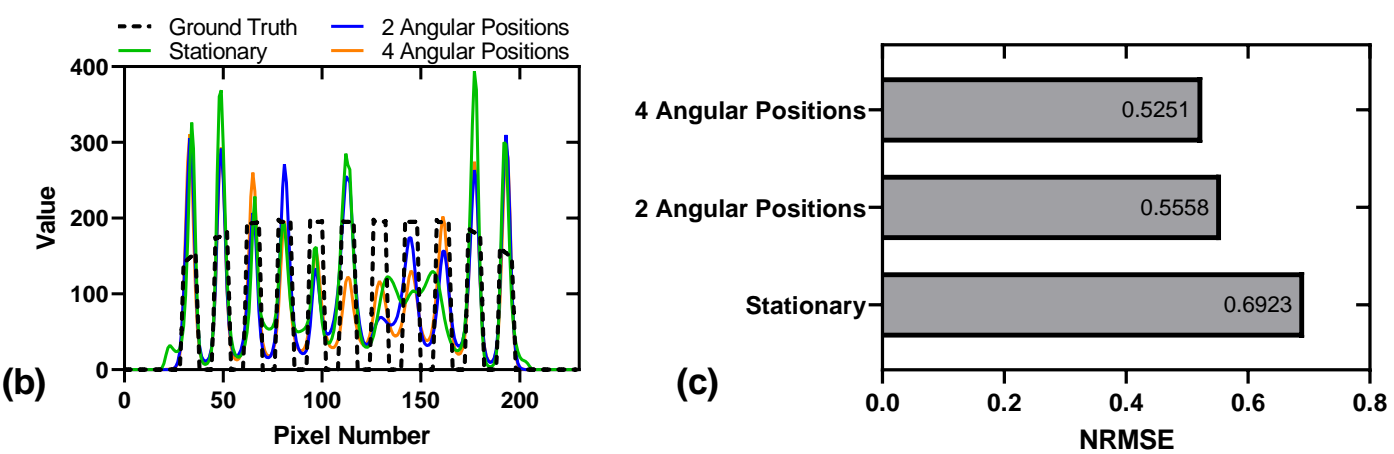

Fig. 5. (a) The mid-transverse slice of the hot-rod phantom's ground truth image and the corresponding reconstructed slices of the phantom at the iterationof-interest for the current prototype design of the AdaptiSPECT-C with a stationary scan, and with two (10 ${ }^{\circ}$ step) and four $\left(5^{\circ}\right.$ step) angular positions. (b) The mid-horizontal profile (along the arrow shown on the ground truth image) of the axially summed slices for the three data acquisition schemes. (c) The NRMSE values at the iteration-of-interest for the three data acquisition schemes. Note although the original hot-rod phantom used in the simulation is cylindrical, the phantom was down-sampled to match the dimensions of the reconstructed image and then image trimming was applied (as described in section II.D) to generate the ground truth image as shown in (a). Notice the improved visibility of the rods and NRMSE improvement in the phantom with increase of angular sampling.

for the disk at the furthest cranial extent) are distinguished. The axial sampling positions with stationary scan are shown by the green arrows in Fig. 6(b). Each axial sampling position contributed in distinguishing 2 nearby disks (totaled 4 disks). Note the axial sampling position corresponding to the QV ring with stationary scan is beyond the axial extent of the phantom and reconstructed image. With two and three axial positions, more axial samples (shown by the blue and orange arrows, respectively, in Fig. 6(b)) are added to those with stationary scan. As seen in Fig. 6(b), the contribution of the additional axial sampling resulted in distinguishing 9 disks. Although an axial sampling is added near the farthest disk at the cranial region with two- and three-axial-position scans, the disk couldn't be distinguished because of the truncation of the VOI happening in that region with the imaging bed translation. The image with three axial positions is noticeably of higher quality and its summed axial profile accordingly shows higher peak-to-valley ratios compared to ones of two axial positions. This trend is in accordance with the substantial improvement in the NRMSE values as plotted in Fig. 6(c) at the iterationof-interest for the stationary (one axial position), two axial positions, and three axial positions. The NRMSE values for stationary, two axial positions, and three axial positions were 0.6360 at iteration 113, 0.4804 at iteration 108, and 0.3984 at iteration 128 , respectively. The volumetric sensitivity over a $21-\mathrm{cm}$ diameter sphere was calculated for the acquisition schemes with one (stationary), two, and three axial positions as $1.00,0.73$, and 0.72 , respectively, relative to the stationary scheme. Non-uniform sampling over the VOI can result in different noise characteristics which is visible in uniform regions of reconstructed images [31]. The CV of the sensitivity value of the voxels within the $21-\mathrm{cm}$ diameter imaging VOI with one (stationary), two, and three axial positions was calculated as $43 \%, 35 \%$, and $34 \%$, respectively, showing more uniform sampling with two and three axial positions compared to the stationary (one axial position) acquisition scheme.

Fig. 7(a) shows sample transverse, coronal, and sagittal slices through the activity distribution of the XCAT phantom simulating the localization of [I-123]-ioflupane DAT imaging agent as being acquired by the stationary prototype system configuration, as well as for augmentations of sampling by the limited detector heads (gantry) rotation(s) and imaging bed translation(s) during data acquisition. For visualization, the image shown here for each sampling scheme is the mean image over the 5 noise realizations after image trimming. The 5 individual noise realizations were used in all calculations of numerical metrics. We down-sampled the XCAT DAT activity phantom to match the dimensions of the reconstructed image. The image trimming was then applied to generate the ground truth image. Notice that although differences can be appreciated in the reconstructed slices visually, especially in striatal regions as well as uniformity in the background, they are less obvious than with imaging of phantoms specifically chosen to test sampling (e.g. the sphere, hot-rod, and Defrise phantoms). Fig. 7 (b) and (c) show the mean NRMSE and SBR values and their standard deviations, respectively, over 5 noise realizations at the iteration-of-interest for the DAT phantom using the stationary configuration and the proposed acquisition 
(a)

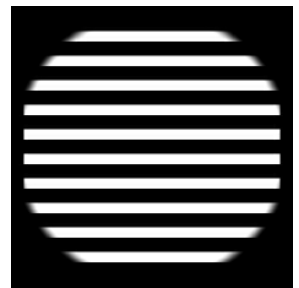

Ground Truth

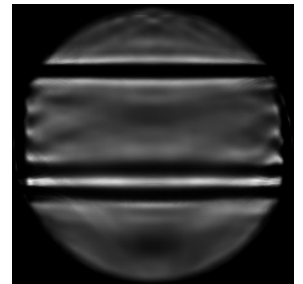

Stationary (1 Axial Position)

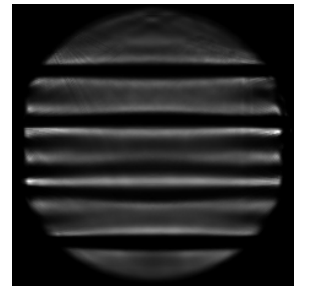

2 Axial Positions

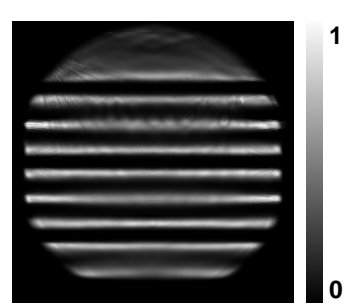

3 Axial Positions
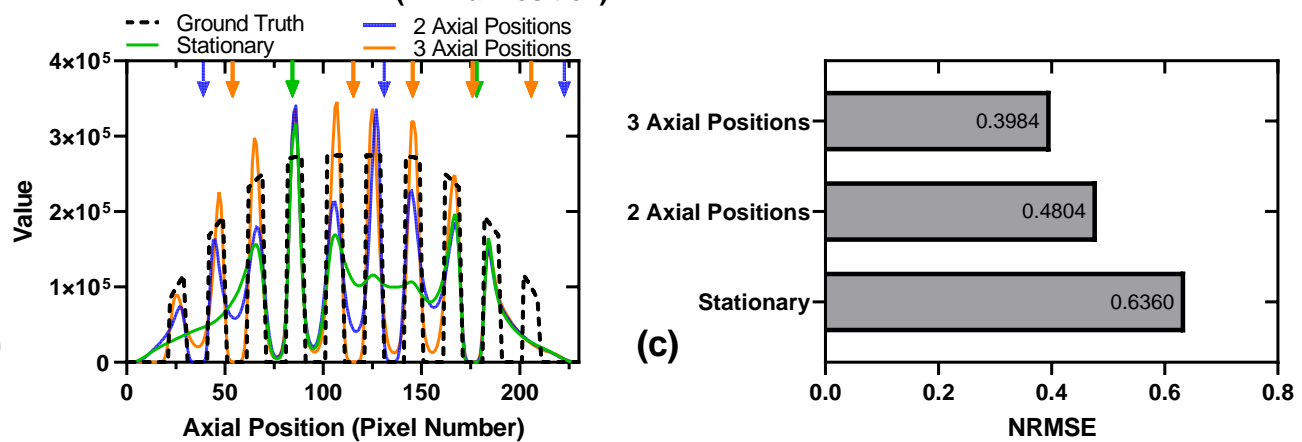

Fig. 6. (a) The mid-coronal slice of the Defrise phantom's ground truth image and the corresponding reconstructed slices of the phantom at the iterationof-interest for the current prototype design of the AdaptiSPECT-C with a stationary scan (one axial position), and with two (4.6 cm step) and three axial positions (3.1 cm steps). (b) The summed axial profile of the Defrise reconstructed images for the three data acquisition schemes obtained by representing each transverse slice by sum of its voxels. (c) The NRMSE values at the iteration-of-interest for the three data acquisition schemes. Note although the original Defrise phantom used in the simulation is cylindrical, the phantom was down-sampled to the dimensions of the reconstructed image and then image trimming was applied (as described in section II.D) to generate the ground truth image in (a) matching the reconstructed images. The axial sampling positions with stationary scan are shown by the green arrows in (b). The axial sampling position corresponding to the QV ring with stationary scan is beyond the axial extent of the phantom and reconstructed image. The axial sampling positions added to the ones of stationary scan with two- and three-axial-position scans because of the imaging bed translation are shown by the blue and orange arrows, respectively, in (b). Although an axial sampling is added near the farthest disk at the cranial region (the rightest region in (b)) with two- and three-axial-position scans, the disk couldn't be distinguished because of the truncation happens in that region with the imaging bed translation. Notice the improved visibility of the disks and NRMSE improvement in the Defrise phantom with increase of axial sampling.

schemes to improve sampling. The iteration-of-interest for the stationary, two and four angular positions, and two and three axial positions was $41,39,38,39$, and 39 , respectively. As seen in Fig. 7(b), the NRMSE values decreased using more angular or axial samples compared to the stationary scheme. The SBR values, as plotted in Fig. 7(c), increased (closer to the true value of 5.33) with enhanced angular or axial sampling.

Similarly, sample slices of the XCAT I-123-IMP brain perfusion ground truth and the corresponding reconstructed slices with the stationary design acquisition together with the proposed approaches (i.e., gantry rotation and imaging bed translation) to augment the sampling are shown in Fig. 8(a). The mean images over the 5 noise realizations after image trimming are shown in Fig. 8. The ground truth image was generated from the brain perfusion phantom using the same approach explained for the DAT phantom before. The corresponding mean NRMSE values over 5 noise realizations with their standard deviations at the iteration-of-interest are plotted in Fig. 8(b). The mean AR(\%) values over 5 noise realizations with their standard deviations for different sampling schemes, at the iteration-of-interest, over 18 different brain regions (Fig. 3) are shown in Fig. 8(c). Note the true value for $\mathrm{AR}(\%)$ is $100 \%$ meaning full recovery of activity in a region. The iteration-of-interest for stationary, two and four angular positions, and two and three axial positions sampling schemes was $36,34,34,35$, and 34, respectively. From Fig. 8(c), the $\mathrm{AR}(\%)$ values of hot regions in the brain perfusion phantom (i.e., gray matter, cerebellum, caudate, putamen, substantia nigra, thalamus, and amygdala) were underestimated which can be due to spilled-out activity. On the other hand, the $\mathrm{AR}(\%)$ values of the cold regions in the phantom (i.e., white matter, corpus callosum, hippocampus, and midbrain) were overestimated in the reconstructed images likely because of spilled-in activity from proximate hot regions. For almost all 18 brain regions, employing more angular or axial sampling enhanced the $\mathrm{AR}(\%)$ values.

In the simulated clinical activity distributions shown in Fig. 7 and 8 , the differences with improved (additional) sampling are less obvious than those observed in the other investigated phantoms. Also, the impact of angular sampling in the clinical simulations can best be noted in the transverse slices while changes due to axial sampling improvement are best appreciated in looking at the axial separation of regions in the coronal and sagittal slices.

Fig. 9(a) shows sample slices of the XCAT I-123-IMP brain perfusion phantom with a $3.2-\mathrm{cm}$-diameter spherical lesion in the right temporal lobe and corresponding reconstructed slices with the stationary acquisition together with the two approaches (i.e., gantry rotation and imaging bed translation) to enhance the sampling. The mean images over the 5 noise 

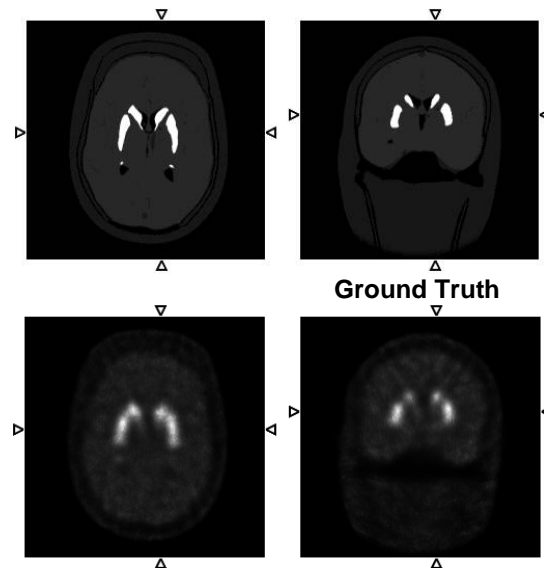

Ground Truth

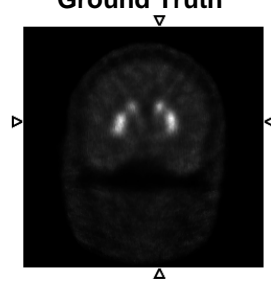

2 Angular Positions
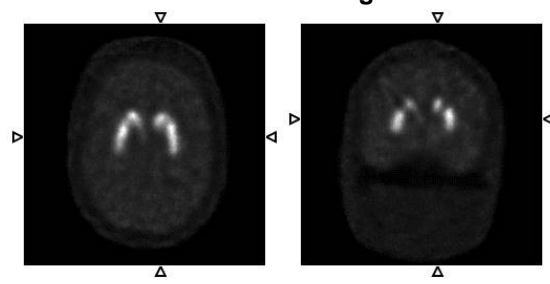

2 Axial Positions
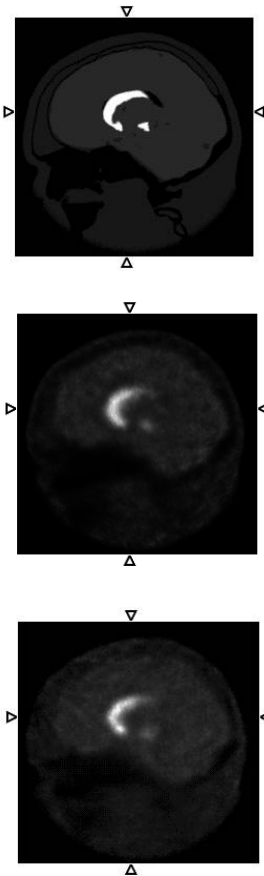

.

(a)
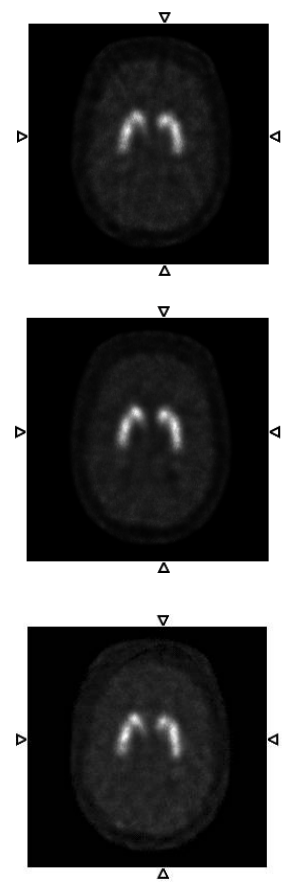

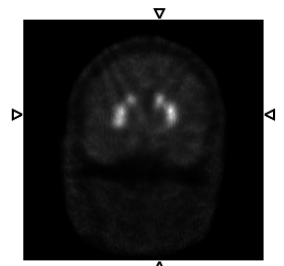

Stationary

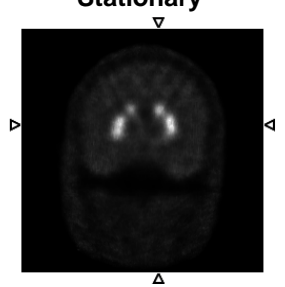

4 Angular Positions

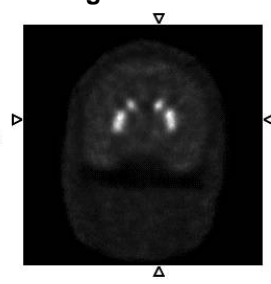

3 Axial Positions
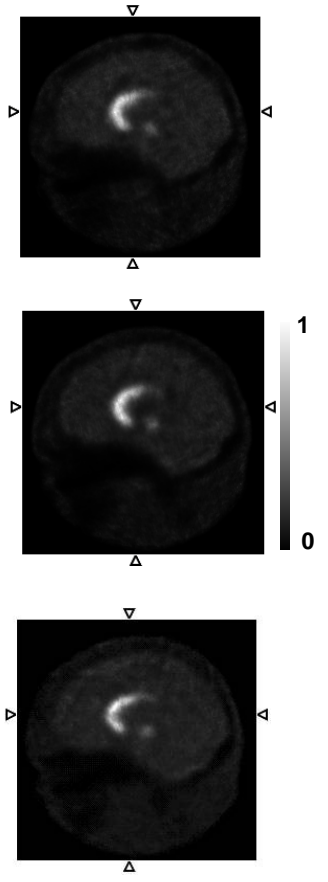

$$
\text { (1) }
$$
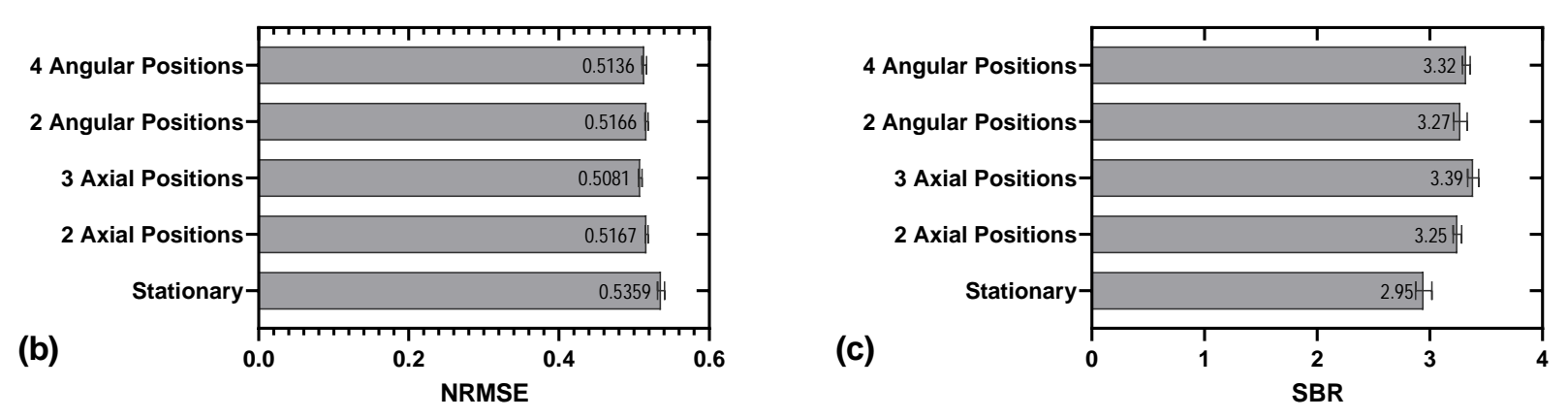

Fig. 7. (a) Sample transverse, coronal, and sagittal slices of the ground truth image of the brain [I-123]-ioflupane DAT phantom together with the corresponding reconstructed slices from simulated acquisition of the phantom by the current prototype design of the AdaptiSPECT-C system with stationary imaging, and using data acquisition schemes with two angular positions of the detector heads in the rings with $10^{\circ}$ azimuthally between, with four angular positions of the heads with three $5^{\circ}$ azimuthal steps, with two axial positions with a $4.6 \mathrm{~cm}$ step, and with three axial positions with $3.1 \mathrm{~cm}$ steps. (b) and (c) The NRMSE and SBR values for the abovementioned cases for the reconstructed images of the DAT phantom at the iteration-of-interest, respectively. The images shown in (a) are the mean image over the 5 noise realizations after image trimming. The NRMSE and SBR values shown in (b) and (c) are the mean values over 5 noise realizations for each sampling scheme. The standard deviation is shown by bar on the plots. The true value for SBR is 5.33. Note subtle changes in visualized detail with alteration in sampling.

realizations after image trimming are shown here. The ground truth image was also generated using the approach explained before from the brain perfusion phantom with the lesion. The mean NRMSE, CRC, bias, and CV values over 5 noise realizations with their standard deviations shown in Fig. 9 (b)-(e), respectively. Note the true value for CRC(\%) is $100 \%$. The CRC and bias values for the 5 sampling scenarios are additionally plotted over iterations of reconstruction in Fig. 9 (f) and (g), respectively, showing that even letting the reconstruction continue after the iteration-of-interest, stationary data acquisition generates images of lower CRC and higher bias. While the plots in Fig. 9 (b)-(e) show slight improvement in the NRMSE, CRC, and CV values at the iteration-of-interest with improved sampling, the bias value at the iteration-of- interest enhances substantially with more angular and axial samples as shown in Fig. 9(d). The iteration-of-interest for the stationary, two and four angular positions, and two and three axial positions were $36,34,33,33$, and 33 , respectively.

\section{B. Contribution of the Quasi-Vertex (QV) Ring of the Detector Heads in Sampling}

Fig. 10(a) shows the reconstructed slices of the Defrise phantom simulated with the current prototype of the AdaptiSPECT-C with and without the QV ring of the detector heads. The iteration-of-interest with and without the QV ring was 113 and 116, respectively. Note the top most region of the images is corresponding to the cranial region of the VOI which is close to the QV ring of the detector heads. No 

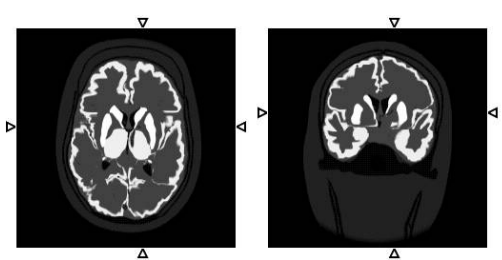

Ground $\stackrel{\Delta}{\nabla}$ ruth
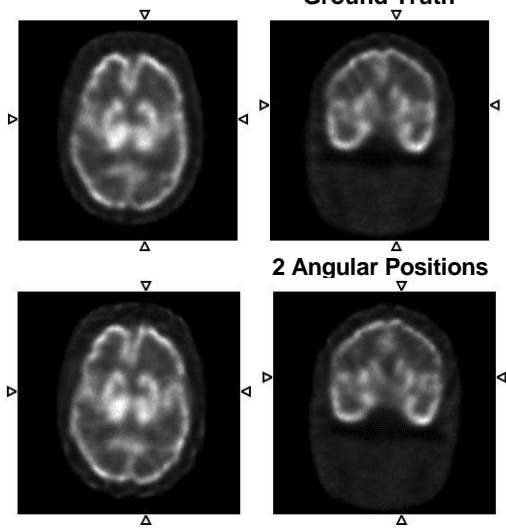

2 Angular Positions

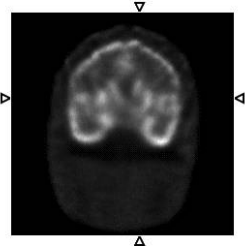

2 Axial Positions
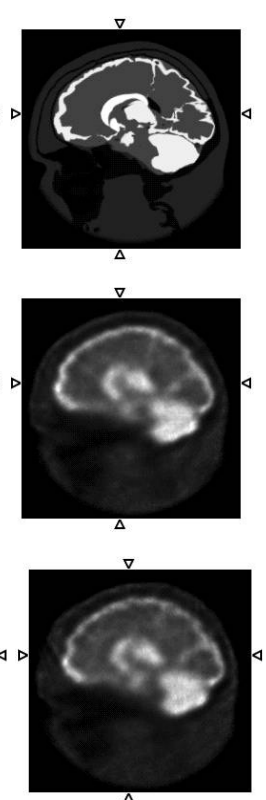

(a)

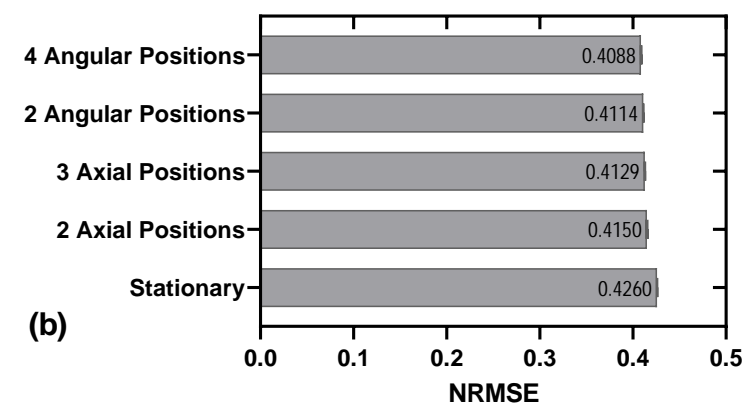

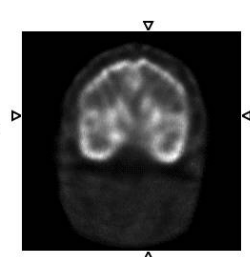
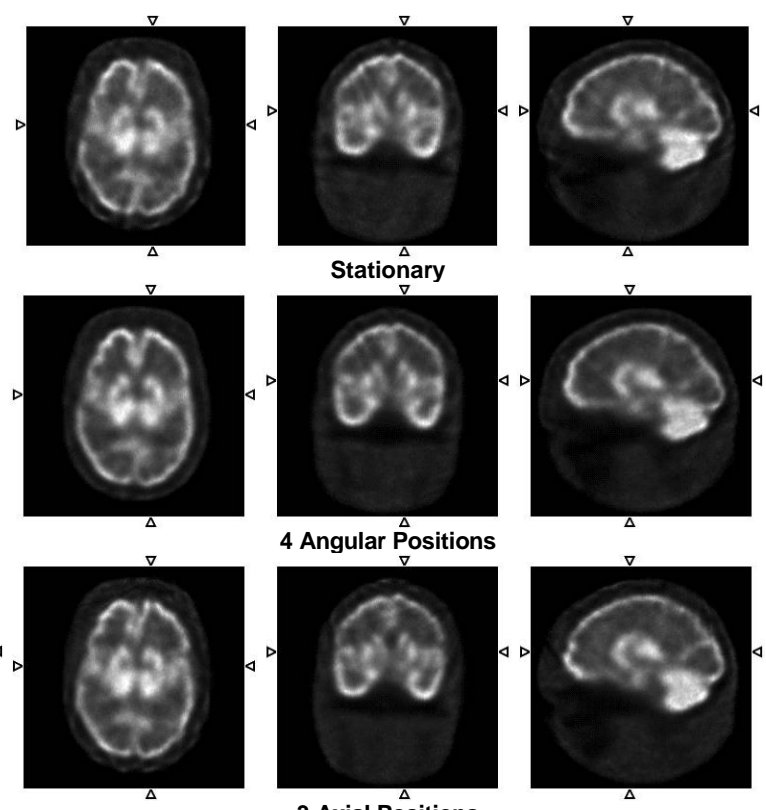

(a)

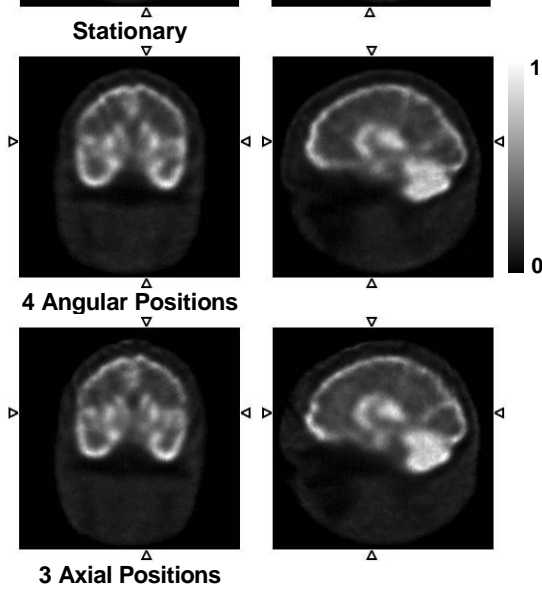

4 Angular Positions

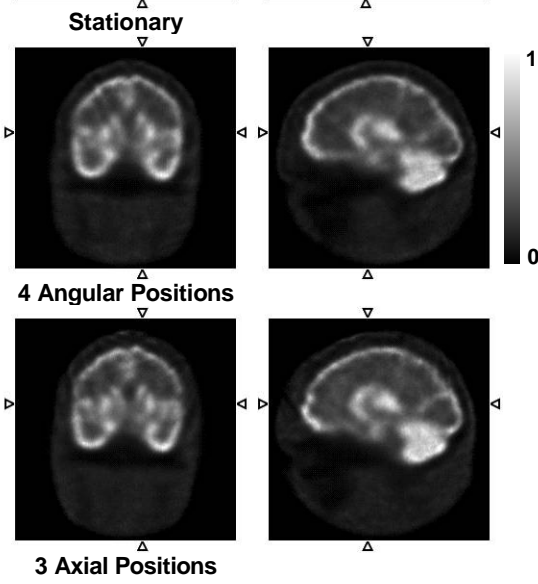

3 Axial Positions
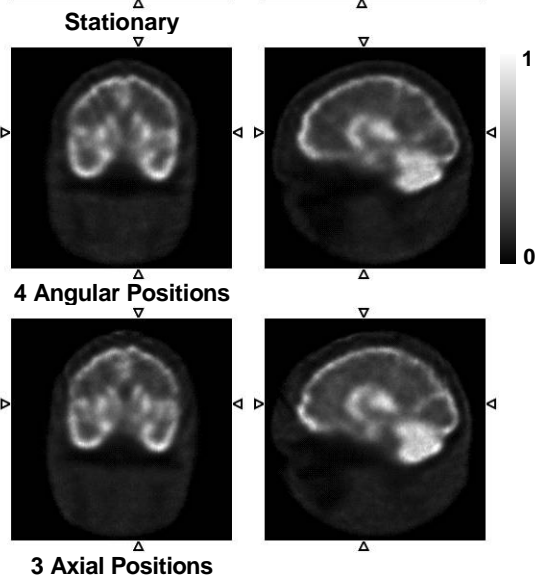

0

.

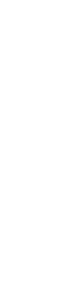



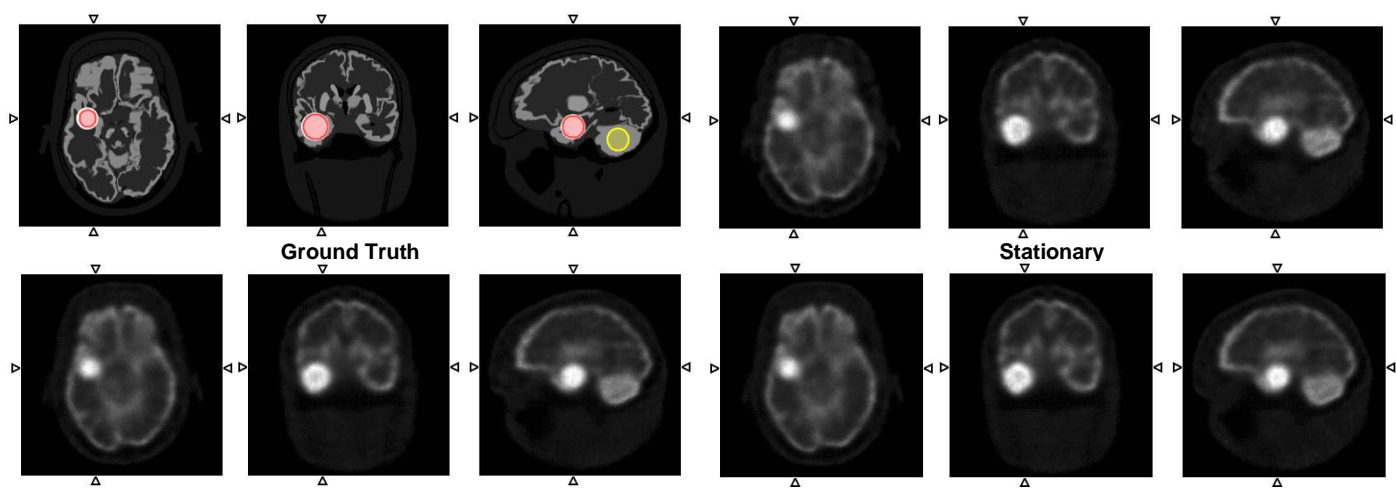

2 Angular Positions
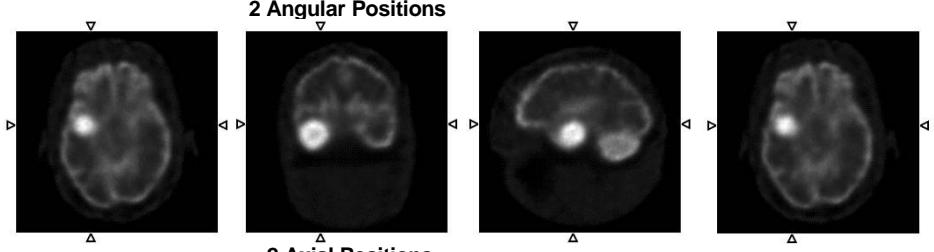

4 Angular Positions

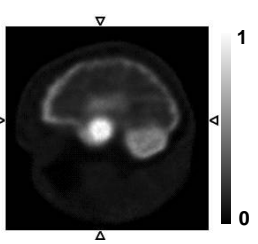

2 Axial Positions

(a)
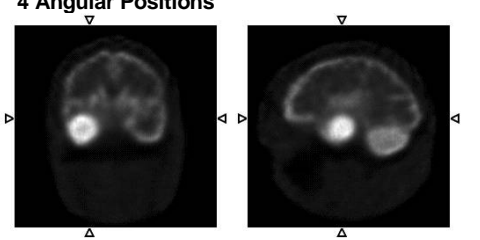

3 Axial Positions
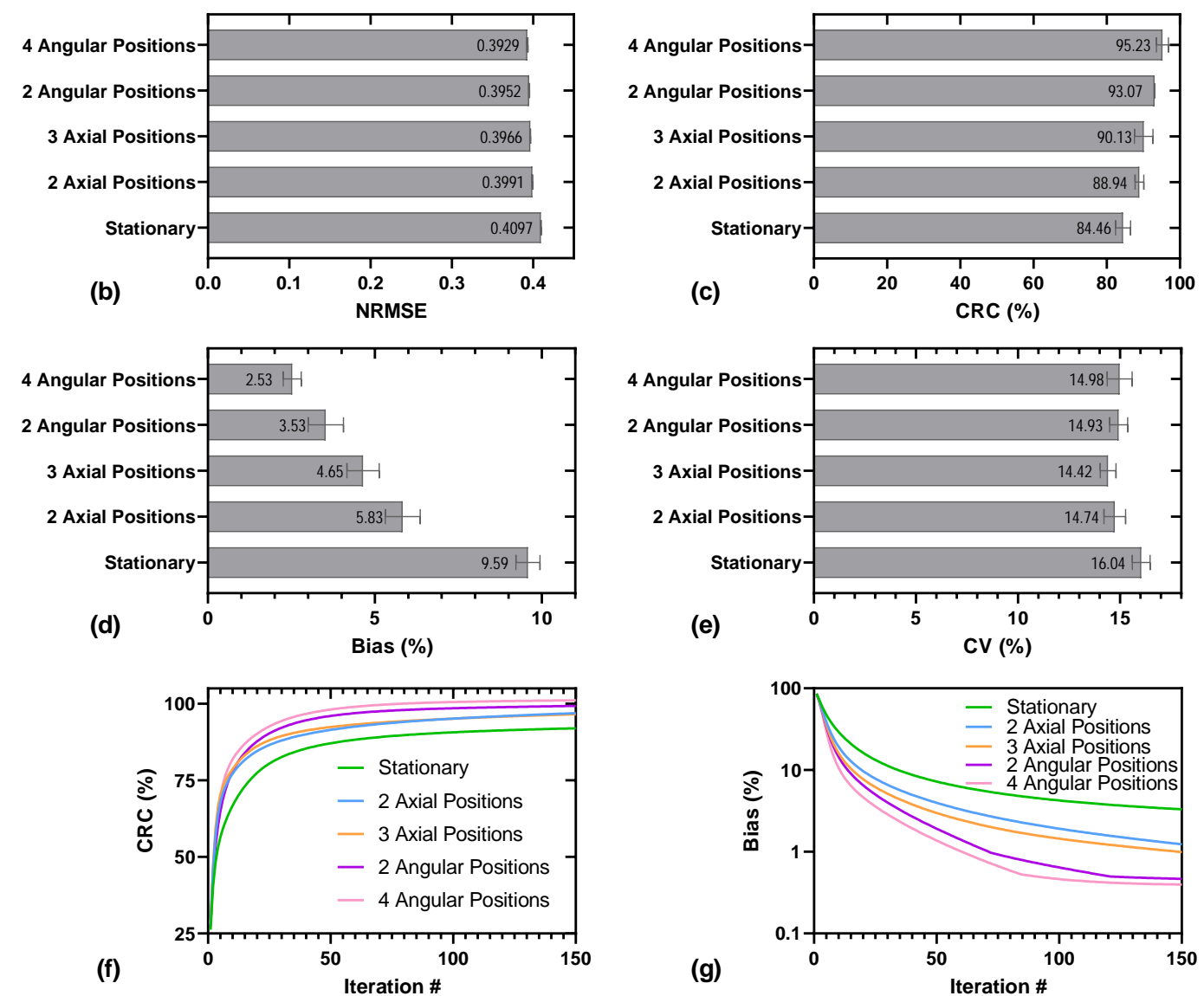

Fig. 9. (a) Sample transverse, coronal, and sagittal slices of the ground truth image of the I-123-IMP brain perfusion phantom with 3.2-cm-diameter spherical lesion in the right temporal lobe are shown together with the corresponding reconstructed slices from simulated acquisition of the phantom by the current prototype design of the AdaptiSPECT-C system with stationary imaging, and using data acquisition scheme with two angular positions of the detector heads in the rings with $10^{\circ}$ azimuthally between, with four angular positions of the heads with three $5^{\circ}$ azimuthal steps, with two axial positions with a $4.6 \mathrm{~cm}$ step, and with three axial positions with $3.1 \mathrm{~cm}$ steps. The spherical VOIs, 0.9 times the size of the original lesion, drawn automatically on the lesion and cerebellum as hot and background VOIs, respectively, to calculate CRC, are also shown (in red and yellow for the hot and background VOI, respectively) on the ground truth image. (b)-(e) The NRMSE, CRC, bias, and CV values for the abovementioned cases for the reconstructed images of the brain perfusion phantom with the lesion at the iteration-of-interest, respectively. (f) and (g) The $\mathrm{CRC}(\%)$ and bias $(\%)$ values for the abovementioned cases over 150 iterations of reconstruction, respectively. The bias $(\%)$ values are shown on a logarithmic axis for better visualization. The images shown in (a) are mean image over the 5 noise realizations after image trimming. The NRMSE, CRC, bias, and CV values shown in (b)-(g) are the mean values over 5 noise realizations for each sampling scheme. The standard deviation is shown by bar on the plots in (b)-(d). The true value for CRC(\%) is $100 \%$. 


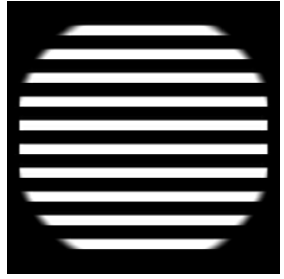

Ground Truth

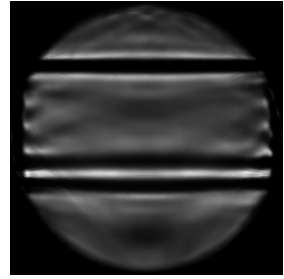

wl QV Ring (a)

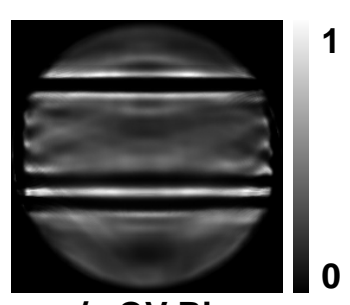

w/o QV Ring

1

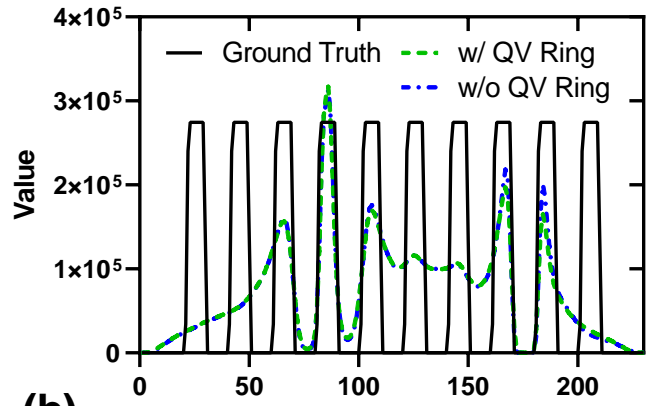

(b)

Axial Position (Pixel Number)
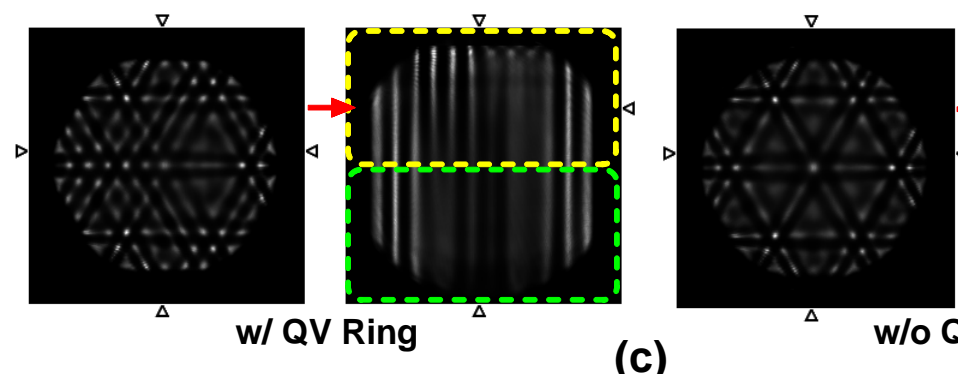

(c)

wlo QV Ring

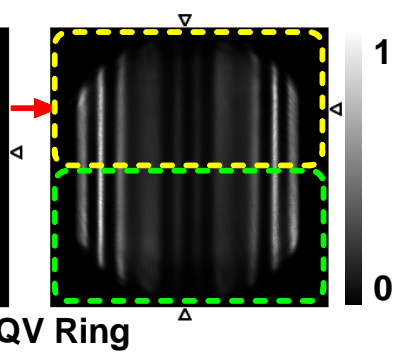

Cranial Hemisphere
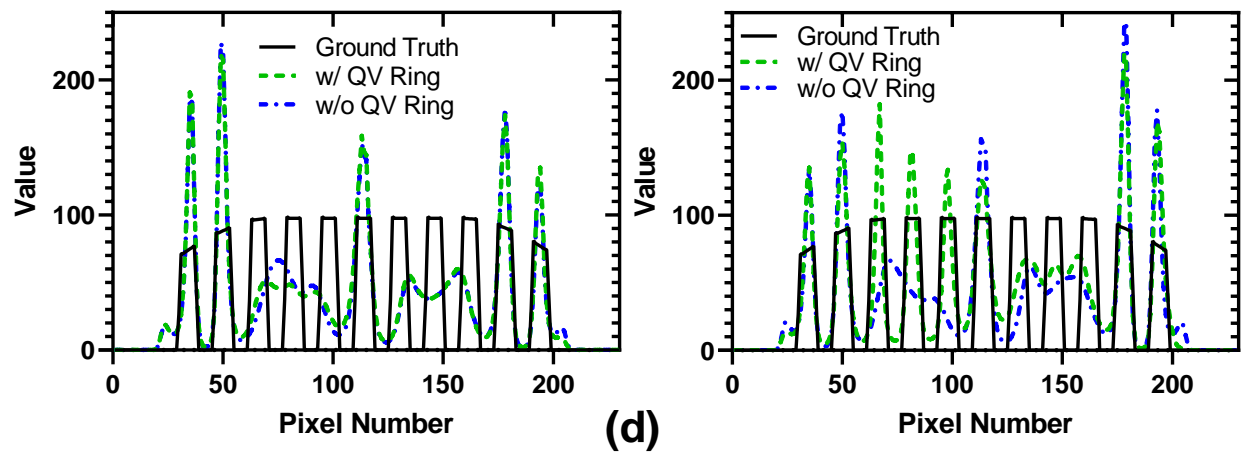

Fig. 10. (a) The mid-coronal slice of the Defrise phantom's ground truth image and the corresponding reconstructed slices at the iteration-of-interest with and without the QV ring of detector heads. (b) The summed axial profile of the Defrise reconstructed images with and without the QV ring of the detector heads obtained by summing the voxels over non-axial directions ( $\mathrm{x}$ and $\mathrm{y}$ ). Note the axial position of the QV ring of the detector heads is toward the top (end) of the shown images (plot). (c) Sample transverse and mid-coronal slices of the reconstructed images of the hot-rod phantom with and without the QV ring of the detector heads. The axial position of the transverse slices is shown on the coronal slices by red arrows. (d) The mid-horizontal profile of the axially summed slices of the hot-rod phantom over the caudal and cranial hemispheres with and without the QV ring. The caudal and cranial hemispheres are shown by green and yellow dashed regions, respectively, on coronal slices in (c).

obvious difference in terms of resolving the hot disks of the Defrise phantom is noticed in the images with and without the QV ring. We plotted the summed axial profile for these two cases in Fig. 10(b) where almost no difference is observed in the summed axial profiles. Based on the performed analytical simulations, the volumetric sensitivity of the AdaptiSPECT$\mathrm{C}$ with the QV rings of the detector heads over the $21-\mathrm{cm}$ spherical VOI is $\sim 1.3$ times higher than that of without the $\mathrm{QV}$ ring. The $\mathrm{CV}$ of the sensitivity value of the voxels within this VOI as a measure of sampling uniformity was $\sim 43 \%$ with and without the QV.

The sample transverse and mid-coronal slices of the reconstructed images of the hot-rod phantoms in Fig. 10(c) simulated with and without the QV ring of the detector heads show the improvement in angular sampling in terms of better rod visualization mainly in the cranial hemisphere of the VOI. As shown in Fig. 10(c), the displayed transverse slice was intentionally chosen to be in the cranial hemisphere to demonstrate the visual improvement. The reconstructed images agree with the mid-horizontal profiles over the axially summed transverse slices calculated separately for the caudal and cranial halves of the image in Fig. 10(d). As shown in Fig. 10(d), the profiles of the caudal and cranial halves are almost the same with and without the QV ring. However, with the QV ring at least three more sharp peaks are observed in the profile of the cranial hemisphere while there is no resolved peak in that region without the QV ring. The reconstruction iteration-of-interest with and without the QV ring was 134. 


\section{Discussion AND CONCLUSIONS}

The current prototype system design of the AdaptiSPECT$\mathrm{C}$ resembles a truncated spherical structure, stationary in nature. In this work, we first studied the axial and angular sampling capability of the stationary prototype system design for the AdaptiSPECT-C. Then, we demonstrated that the reconstructed images of the AdaptiSPECT-C can be enhanced, qualitatively and quantitatively, by augmenting the angular and axial sampling. For this, two approaches were proposed and then evaluated for improving the sampling capability of the base prototype design using gantry rotation and imaging bed translation. In these investigations, an in-house fast system modeling approach [40] was used for both simulations and iterative reconstructions.

We investigated two augmented axial sampling acquisition schemes by adding axial shifts of the source distribution (translation of patient imaging bed). The first consisted of adding one axial bed translation to the base position and the second adding two translations. In each case, the translations were selected based on acquiring evenly distributed axial samples over the imaging VOI. Also, when reconstructing the data acquired with axial shifts, all the acquired projection data were reconstructed, simultaneously. This technique results in images of higher quality compared to separate reconstruction of image volumes corresponding to acquisition steps and then combining them [49]. Further, as illustrated in Fig. 6 (a) and (b), when we investigated axial sampling for the current stationary prototype system design for AdaptiSPECT-C, we determined it was unable to resolve more than 4 of the 10 disks in the Defrise phantom. By simulating one- and two-stage imaging bed translation (i.e., two and three axial positions, respectively), 9 of the 10 disks (i.e., all disks except the one at the furthest cranial extent) are distinguishable in the reconstructed image and there was a $24 \%$ and $37 \%$ decrease in the NRMSE with two and three axial positions, respectively (Fig. 6(c)). For the XCAT phantom with the simulated I-123IMP brain perfusion distribution, the change in the NRMSE with simulated bed translation was a $<3 \%$ decrease (Fig. 8(b)) although the changes in the appearance of the slices was visible as improved quality with increased axial positions (Fig. 8(a)). The AR(\%) values, defined as a task-based metric of quantification, showed improvement with two and three axial positions compared to stationary data acquisition for all the brain regions of high activity concentration (except for the substantia nigra) including gray matter, cerebellum, caudate, putamen, thalamus, and amygdala. Although substantia nigra is sampled by all pinholes, the partial volume effect (PVE) [50] due to its small size $(\sim 0.6 \mathrm{ml})$ seems to impact the way the region is being reconstructed. The $\operatorname{AR}(\%)$ values for the brain regions of low activity concentration don't follow the same trend. While the $\mathrm{AR}(\%)$ improve for white matter, corpus callosum, and medulla oblangata with more axial positions, no improvement is observed for hippocampus and midbrain. For the brain phantom with the simulated activity distribution of I-123-ioflupane DAT agent, the NRMSE changes $<5 \%$ with more axial positions (Fig. 7(a)). The SBR was improved by $10 \%$ and $15 \%$, compared to the stationary scheme, using two and three axial positions, respectively, moving closer to the simulated true value of 5.33 (Fig. 7(c)). The reconstructed slices of the DAT phantom show higher resolution in the striatal region, better caudate and putamen differentiation, and more uniform background with more axial positions. The reconstructed slices of the simulated XCAT I-123-IMP brain perfusion phantom with the lesion showed higher quality for both the lesion site and other brain regions with two and three axial positions (Fig. 9(a)). In summary, the simulations using the XCAT brain perfusion and DAT phantoms with stationary scan versus with two and three axial bed positions (Fig. 7-9) show an enhancement in the quality of the reconstructed images, but as one would expect less dramatic changes than with the Defrise phantom which is designed to challenge axial sampling.

In general, for each system geometry, the optimum number of angular views, as well as theoretical considerations depends on the activity distribution, imaging task, and the availability of prior information $[31,51]$. In the current system design, the detector heads in the caudal, middle, and QV rings contribute in angular sampling. We simulated limited rotation of the current detector heads by two approaches to evaluate the effect of more angular views for the prototype system design. In the first approach, we simulated a single rotation of $10^{\circ}$ which doubled the number of angular views providing angular sampling at $10^{\circ}$ azimuthal intervals. In the second approach, we simulated a sequence of three $5^{\circ}$ rotations which quadruples the number of angular views providing angular samplings at $5^{\circ}$ azimuthal intervals. According to Fig. 4(a), substantial enhancement is seen in the uniformity of the reconstructed slices of the sphere phantom both visually and quantitatively with a single $10^{\circ}$ rotation of the system. The non-uniformity, NRMSE, bias, and CV values (Fig. 4 (b)-(e)) agree with the visual enhancement; however, no noticeable quantitative improvement is seen with the increase to 4 angular positions as compared to 2 angular positions. Also, Fig. 5(a) shows how the spatial resolution at different regions of the transverse FOV is improved with two and four angular positions compared to stationary data acquisition. While the image with stationary scheme has the lowest quality among the three schemes, almost all the hot rods are resolved with four angular positions. The NRMSE values decreased by $20 \%$ and $24 \%$ with two and four angular positions, respectively. Similar trends are seen with the reconstructed slices of the brain DAT and perfusion phantoms (Fig. 7-9). Higher resolution in the striatal region, leading towards better differentiation of the caudate and putamen. Also, better uniformity in the background activity in the brain is noticed in the reconstructed slices of the DAT phantom with more angular samples (Fig. 7(a)). The NRMSE values decreased by $\sim 4 \%$ with more (i.e., two and four) angular positions (Fig. 7(b)). The SBR values increased by $11 \%$ and $12 \%$ with two and four angular positions, respectively (Fig. 7(c)). The decrease of $3 \%$ and $4 \%$ in the NRMSE values with two and four angular positions, respectively, in the brain perfusion images is in accordance with higher image quality of the reconstructed slices with more angular samples (Fig. 8 (a) and (b)). The same impact results in better activity recovery for all the assessed hot regions in the brain perfusion phantom except for the 
substantia nigra (Fig. 8(c)). As described earlier, quantification in substantia nigra with its small size seems to be limited due to PVE. More angular samples resulted in images of higher quality for the brain perfusion phantom with the lesion observed as better reconstruction of tumor boundaries and activity uniformity in addition to better resolution for other brain regions (Fig. 9(a)). The image NRMSE, and the lesion CRC, bias, and CV improved by $3 \%$ (4\%), $10 \%$ (13\%), $63 \%$ (74\%), and $7 \%(7 \%)$ with two angular positions (with four angular positions), (Fig. 9 (b)-(e)). The CRC(\%) of the lesion at higher iterations approaches the true value of $100 \%$ with more angular samples (Fig. 9(f)). In summary, for the XCAT brain DAT and perfusion simulations by all measures, a 2fold increase in angular sampling is very desirable; however, a 4-fold increase likely is not needed.

As part of a separate study [28, 52] we investigated the impact of photons emitted in structures throughout the body, and detected by the QV and other rings of AdaptiSPECT-C. In this work we assessed the contribution of the QV ring of the detector heads in angular and axial sampling. The results (Fig. 10 (a) and (b)) confirm that in the stationary system design only the apertures in the middle and caudal rings contribute to the axial sampling. The additional detector heads in the QV ring do increase the sensitivity of the system and provide more information which aids reconstruction at no extra cost in imaging time. Also, the reconstructed slices of the hotrod phantom with the QV ring are of better spatial resolution showing the contribution of the QV ring of the detector heads in angular sampling, mainly for the cranial hemisphere of the imaging VOI (Fig. 10 (c) and (d)).

Notice that adding more rings of detector heads and/or more detector heads per ring can increase the number of axial and/or angular samples, respectively. However, in addition to the manufacturing cost escalation, the additional detector heads of the same size cannot fit in the same truncated spherical geometry (see Fig. 1) unless they are arranged at a farther distance from the imaging VOI. With the same imaging VOI but with detectors at a farther distance from it, the aperture positions should also be repositioned to farther from the imaging VOI and closer to the detectors to avoid truncation of projection image. Moving the apertures to farther from the imaging VOI worsens both spatial resolution and sensitivity. To maintain spatial resolution, the aperture diameters could be reduced in size, but this would further decrease sensitivity. To some extent, this loss of sensitivity can be balanced by the increase in the number of pinholes and detectors acquiring data. Finding the optimum combination of these parameters when designing a SPECT system dedicated to brain imaging has been excellently investigated previously by others [53, 54], and thus will not be discussed further in this paper on the sampling aspects of SPECT brain imaging with pinhole collimation.

This study showed in the phantoms specifically designed to investigate sampling issues that the axial and angular sampling of the current system design is inadequate, especially in regions corresponding to the caudal hemisphere of the imaging VOI. Still the images of the simulated brain perfusion (with and without the lesion) and DAT phantoms show that even the current stationary system design with no additional axial or angular sampling can reconstruct good-quality images. However, the results demonstrate that enhancing this current base axial and angular sampling can further improve image quality. Nevertheless, using axial translation of the imaging bed in the AdaptiSPECT-C to enhance the axial sampling has some drawbacks. Translation of the bed during the scan can cause patient motion different than the bed which would necessitate motion tracking and compensation during reconstruction. More importantly, according to our simulations, the volumetric sensitivity of the system, as defined in section II.E, drops $\sim 28 \%$ with the simulated bed translations due to the truncation happening in cranial regions with these axial translations. On the other hand, the usage of gantry rotation to augment angular sampling would likely introduce additional design complexity, safety issues, and cost. Also, there is overhead time associated with both types of movements. Note gantry rotation and/or imaging bed translation degrade the system's capability of dynamic imaging. Therefore, we have been exploring alternative approaches for enhancing axial and angular sampling. We intend to take advantage of introducing more axial and/or angular samples to our base design by using additional apertures for each detector head with shuttering of apertures for temporal control of extent of multiplexing [5557].

We started with a stationary imaging system for brain imaging and determined ways in which imaging performance of such a system may be improved by increasing angular and axial sampling. We also evaluated the image quality difference with different sampling schemes using various metrics. Based on the simulations performed for investigation of different axial sampling schemes, the scheme with three axial positions resulted in the best images for the I-123-IMP brain perfusion (with and without the lesion) and the I-123-ioflupane DAT phantoms. It decreased the NRMSE by $3 \%$ compared to the stationary scheme for the brain perfusion phantom without the lesion. For the brain perfusion phantom with the lesion, the image NRMSE, and the lesion CRC, bias, and CV were improved compared to the stationary scheme by $3 \%, 7 \%, 52 \%$, and $10 \%$, respectively, with three axial positions. For the DAT phantom, the NRMSE and SBR were improved compared to the stationary scheme by $5 \%$ and $15 \%$, respectively, using three axial positions. According to the simulations conducted for investigation of different angular sampling schemes, the best images for the brain perfusion (with and without the lesion) and DAT phantoms were obtained by the scheme with four angular positions. For the brain perfusion phantom without the lesion, the NRMSE decreased by $4 \%$ compared to the stationary scheme using four angular positions. For the brain perfusion phantom with the lesion, the image NRMSE, and the lesion $\mathrm{CRC}$, bias, and $\mathrm{CV}$ were enhanced compared to the stationary scheme by $4 \%, 13 \%, 74 \%$, and $7 \%$, respectively, with four angular positions. Such relative enhancement for the NRMSE and SBR values in the DAT phantom's images were $4 \%$ and $12 \%$, respectively. More improvement in image quality was obtained with going from the first (i.e., stationary scheme) to the second level of increased sampling (i.e., two axial or angular positions), than from the second to the final 
level of sampling (i.e., three axial or four angular positions). We therefore believe although further increases in sampling positions would result in further sampling enhancement, with the increased cost in lost sensitivity during bed/gantry movement and system design complexity, it would possibly not be advantageous overall. Our findings herein have helped us understand what issues need to be addressed in the future through investigation of temporal shuttering of multiple pinholes for each detector head as an alternative for rotation of the system and translation of the imaging bed, data multiplexing, motion tracking and compensation, task performance evaluation, and human observer studies.

\section{ACKNOWLEDGMENT}

Some preliminary results of this work were partially published in the proceedings of IEEE NSS/MIC 2017 by Zeraatkar et al. as "Preliminary Investigation of Axial and Angular Sampling in Multi-Pinhole AdaptiSPECT-C with XCAT Phantoms." The authors would like to thank Dr. Justin C. Goding for proofreading of the manuscript. We also express our gratitude to the reviewers for their suggestions addressing of which enhanced this article.

\section{REFERENCES}

[1] S. G. Hasselbalch, G. Oberg, S. A. Sorensen, A. R. Andersen, G. Waldemar, J. F. Schmidt et al., "Reduced regional cerebral blood flow in Huntington's disease studied by SPECT," J Neurol Neurosurg Psychiatry, vol. 55, no. 11, pp. 1018-23, Nov 1992.

[2] N. C. Reynolds, Jr., R. S. Hellman, R. S. Tikofsky, R. W. Prost, L. P. Mark, B. R. Elejalde et al., "Single photon emission computerized tomography (SPECT) in detecting neurodegeneration in Huntington's disease," Nucl Med Commun, vol. 23, no. 1, pp. 13-8, Jan 2002.

[3] P. Scheltens, "Imaging in Alzheimer's disease," Dialogues Clin Neurosci, vol. 11, no. 2, pp. 191-9, 2009.

[4] L. Binkovitz and P. Thacker, "What does molecular imaging reveal about the causes of ADHD and the potential for better management?," Current Psychiatry, vol. 14, no. 9, pp. 34-42, 2015.

[5] S. R. Suwijn, C. J. van Boheemen, R. J. de Haan, G. Tissingh, J. Booij and R. M. de Bie, "The diagnostic accuracy of dopamine transporter SPECT imaging to detect nigrostriatal cell loss in patients with Parkinson's disease or clinically uncertain parkinsonism: a systematic review," EJNMMI Research, vol. 5, no. 12, 2015.

[6] P. Jensen, L. Feng, I. Law, C. Svarer, G. M. Knudsen, J. D. Mikkelsen et al., "TSPO Imaging in Glioblastoma Multiforme: A Direct Comparison Between 123I-CLINDE SPECT, 18F-FET PET, and GadoliniumEnhanced MR Imaging," J Nucl Med, vol. 56, no. 9, pp. 1386-90, Sep 2015.

[7] K. P. Banks, J. G. Peacock, M. N. Clemenshaw and P. H. Kuo, "Optimizing the Diagnosis of Parkinsonian Syndromes With (123)IIoflupane Brain SPECT," AJR Am J Roentgenol, pp. 1-11, Apr 172019.

[8] I. G. Zubal, S. S. Spencer, K. Imam, J. Seibyl, E. O. Smith, G. Wisniewski et al., "Difference images calculated from ictal and interictal technetium-99m-HMPAO SPECT scans of epilepsy," J Nucl Med, vol. 36, no. 4, pp. 684-9, Apr 1995.

[9] K. A. McNally, A. L. Paige, G. Varghese, H. Zhang, E. J. Novotny, Jr., S. S. Spencer et al., "Localizing value of ictal-interictal SPECT analyzed by SPM (ISAS)," Epilepsia, vol. 46, no. 9, pp. 1450-64, Sep 2005.

[10] T. Abraham and J. Feng, "Evolution of brain imaging instrumentation," Semin Nucl Med, vol. 41, no. 3, pp. 202-19, May 2011.

[11] D. E. Kuhl, R. Q. Edwards, A. R. Ricci, R. J. Yacob, T. J. Mich and A. Alavi, "The Mark IV system for radionuclide computed tomography of the brain," Radiology, vol. 121, no. 2, pp. 405-13, Nov 1976.

[12] K. Kouris, D. C. Costa, P. H. Jarritt, C. E. Townsend and P. J. Ell, "Brain SPECT Using a Dedicated Three-Headed Camera System," Journal of Nuclear Medicine Technology, vol. 20, no. 2, pp. 68-72, 1992.

[13] R. K. Rowe, J. N. Aarsvold, H. H. Barrett, J. C. Chen, W. P. Klein, B. A. Moore et al., "A stationary hemispherical SPECT imager for threedimensional brain imaging," J Nucl Med, vol. 34, no. 3, pp. 474-80, Mar 1993.
[14] F. Zito, A. Savi and F. Fazio, "CERASPECT: a brain-dedicated SPECT system. Performance evaluation and comparison with the rotating gamma camera," Phys Med Biol, vol. 38, pp. 1433-1442, 1993.

[15] W. F. Sensakovic, M. C. Hough and E. A. Kimbley, "ACR testing of a dedicated head SPECT unit," J Appl Clin Med Phys, vol. 15, no. 4, p. 4632, Jul 82014.

[16] F. J. Beekman, F. van der Have, M. C. Goorden, P. E. B. Vaissier, J. van Roosmalen, H. During et al., "G-SPECT-I: a full ring high sensitivity and ultra-fast clinical molecular imaging system with $<3 \mathrm{~mm}$ resolution," in EANM, 2015, vol. 42, no. S209: European Journal of Nuclear Medicine and Molecular Imaging.

[17] Y. Chen, B. Vastenhouw, C. Wu, M. C. Goorden and F. J. Beekman, "Optimized image acquisition for dopamine transporter imaging with ultra-high resolution clinical pinhole SPECT," Phys Med Biol, vol. 63, no. 22, p. 225002, Nov 72018.

[18] P. D. Esser, P. O. Alderson, R. J. Mitnick and J. J. Arliss, "Angledcollimator SPECT (A-SPECT): an improved approach to cranial single photon emission tomography," J Nucl Med, vol. 25, no. 7, pp. 805-9, Jul 1984.

[19] P. D. Esser, "Improvements in SPECT technology for cerebral imaging," Semin Nucl Med, vol. 15, no. 4, pp. 335-46, Oct 1985.

[20] B. M. Tsui, G. T. Gullberg, E. R. Edgerton, D. R. Gilland, J. R. Perry and W. H. McCartney, "Design and clinical utility of a fan beam collimator for SPECT imaging of the head," J Nucl Med, vol. 27, no. 6, pp. 810-9, Jun 1986.

[21] G. T. Gullberg, G. L. Zeng, F. L. Datz, P. E. Christian, C. H. Tung and H. T. Morgan, "Review of convergent beam tomography in single photon emission computed tomography," Phys Med Biol, vol. 37, no. 3, pp. 507-34, Mar 1992.

[22] T. C. Lee, J. R. Ellin, Q. Huang, U. Shrestha, G. T. Gullberg and Y. Seo, "Multipinhole collimator with 20 apertures for a brain SPECT application," Med Phys, vol. 41, no. 11, p. 112501, Nov 2014.

[23] K. Ogawa and Y. Ichimura, "Simulation study on a stationary data acquisition SPECT system with multi-pinhole collimators attached to a triple-head gamma camera system," Ann Nucl Med, vol. 28, no. 8, pp. 716-24, Oct 2014.

[24] M. A. King, J. M. Mukherjee, A. Könik, I. G. Zubal, J. Dey and R. Licho, ”Design of a Multi-Pinhole Collimator for I-123 DaTscan Imaging on Dual-Headed SPECT Systems in Combination with a FanBeam Collimator," IEEE Transactions on Nuclear Science, vol. 63, no. 1, pp. 90-97, 2016.

[25] L. Chen, B. M. W. Tsui and G. S. P. Mok, "Design and evaluation of two multi-pinhole collimators for brain SPECT," Ann Nucl Med, vol. 31, no. 8, pp. 636-648, Oct 2017.

[26] A. Könik, J. D. Beenhouwer, J. M. Mukherjee, K. Kalluri, S. Banerjee, N. Zeraatkar et al., "Simulations of a Multipinhole SPECT Collimator for Clinical Dopamine Transporter (DAT) Imaging," IEEE Transactions on Radiation and Plasma Medical Sciences, vol. 2, no. 5, pp. 444-451, 2018.

[27] N. Zeraatkar, K. S. Kalluri, A. Könik, J. M. Mukherjee, J. Dey, J. C. Goding et al., "Preliminary Investigation of Axial and Angular Sampling in Multi-Pinhole AdaptiSPECT-C with XCAT Phantoms," in IEEE NSS/MIC, Atlanta, GA, 2017.

[28] J. C. Goding, K. S. Kalluri, A. Könik, Y. He, G. Zubal, L. R. Furenlid et al., "An Investigation of Quasi-Vertex Views in Brain SPECT ImagingInitial Results," in IEEE NSS/MIC, Atlanta, GA, 2017.

[29] N. C. Momsen, G. Richards, M. A. King, N. Zeraatkar and L. R. Furenlid, "Design of a Selectable Pinhole Module for Use in Adaptive SPECT Collimators," in IEEE NSS/MIC, Sydney, Australia, 2018.

[30] H. K. Tuy, "An Inversion Formula for Cone-Beam Reconstruction," SIAM Journal on Applied Mathematics, vol. 43, no. 3, pp. 546-552, 1983.

[31] K. Van Audenhaege, R. Van Holen, S. Vandenberghe, C. Vanhove, S. D. Metzler and S. C. Moore, "Review of SPECT collimator selection, optimization, and fabrication for clinical and preclinical imaging," Med Phys, vol. 42, no. 8, pp. 4796-813, Aug 2015.

[32] A. Poston, "Human Engineering Design Data Digest," ed: Department of Defense Human Factors Engineering Technical Advisory Group, 2000.

[33] W. P. Segars, G. Sturgeon, S. Mendonca, J. Grimes and B. M. Tsui, "4D XCAT phantom for multimodality imaging research," Med Phys, vol. 37, no. 9, pp. 4902-15, Sep 2010.

[34] H. Ito, T. Sato, H. Odagiri, K. Inoue, M. Shidahara, T. Suhara et al., "Brain and whole body distribution of N-isopropyl-4-iodoamphetamine (I-123) in humans: comparison of radiopharmaceuticals marketed by different companies in Japan," Ann Nucl Med, vol. 20, no. 7, pp. 493-8, Aug 2006. 
[35] I. Sarikaya, "PET studies in epilepsy," Am J Nucl Med Mol Imaging, vol. 5, no. 5, pp. 416-30, 2015

[36] J. C. Dickson, L. Tossici-Bolt, T. Sera, K. Erlandsson, A. Varrone, K Tatsch et al., "The impact of reconstruction method on the quantification of DaTSCAN images," Eur J Nucl Med Mol Imaging, vol. 37, no. 1, pp. 23-35, Jan 2010.

[37] C. McArthur, R. Jampana, J. Patterson and D. Hadley, "Applications of cerebral SPECT," Clin Radiol, vol. 66, no. 7, pp. 651-61, Jul 2011.

[38] T. A. Soderlund, J. C. Dickson, E. Prvulovich, S. Ben-Haim, P. Kemp, J. Booij et al., "Value of semiquantitative analysis for clinical reporting of 123I-2-beta-carbomethoxy-3beta-(4-iodophenyl)-N-(3fluoropropyl)nortropane SPECT studies," J Nucl Med, vol. 54, no. 5, pp. 714-22, May 2013.

[39] P. H. Kuo, R. Avery, E. Krupinski, H. Lei, A. Bauer, S. Sherman et al., "Receiver-operating-characteristic analysis of an automated program for analyzing striatal uptake of 123I-ioflupane SPECT images: calibration using visual reads," J Nucl Med Technol, vol. 41, no. 1, pp. 26-31, Mar 2013.

[40] N. Zeraatkar, B. Auer, K. Kalluri, L. R. Furenlid, P. H. Kuo and M. A. King, "GPU-accelerated generic analytic simulation and image reconstruction platform for multi-pinhole SPECT systems", Proc. SPIE 11072, 15th International Meeting on Fully Three-Dimensional Image Reconstruction in Radiology and Nuclear Medicine, 1107216 (28 May 2019).

[41] Z. Huaxia and A. J. Reader, "Fast ray-tracing technique to calculate line integral paths in voxel arrays," in 2003 IEEE Nuclear Science Symposium. Conference Record (IEEE Cat. No.03CH37515), 2003, vol. 4, pp. 2808-2812 Vol.4

[42] L. A. Shepp and Y. Vardi, "Maximum likelihood reconstruction for emission tomography," IEEE Trans Med Imaging, vol. 1, no. 2, pp. 113-22, 1982.

[43] K. Lange and J. A. Fessler, "Globally convergent algorithms for maximum a posteriori transmission tomography," IEEE Transactions on Image Processing, vol. 4, no. 10, pp. 1430-1438, 1995.

[44] C. E. Shannon, "Communication in the Presence of Noise," Proceedings of the IRE, vol. 37, no. 1, pp. 10-21, 1949.

[45] H. H. Barret and K. J. Myers, Foundations of Image Science. Hoboken: John Wiley \& Sons, 2004.

[46] S. T. Mahmood, K. Erlandsson, I. Cullum and B. F. Hutton, "The potential for mixed multiplexed and non-multiplexed data to improve the reconstruction quality of a multi-slit-slat collimator SPECT system," Phys Med Biol, vol. 55, no. 8, pp. 2247-68, Apr 212010

[47] D. S. Djang, M. J. Janssen, N. Bohnen, J. Booij, T. A. Henderson, K. Herholz et al., "SNM practice guideline for dopamine transporter imaging with 123I-ioflupane SPECT 1.0," J Nucl Med, vol. 53, no. 1, pp. 154-63, Jan 2012.

[48] J. E. Juni, A. D. Waxman, M. D. Devous, Sr., R. S. Tikofsky, M. Ichise, R. L. Van Heertum et al., "Procedure guideline for brain perfusion SPECT using (99m)Tc radiopharmaceuticals 3.0," J Nucl Med Technol, vol. 37, no. 3, pp. 191-5, Sep 2009.

[49] B. Vastenhouw and F. Beekman, "Submillimeter total-body murine imaging with U-SPECT-I," J Nucl Med, vol. 48, no. 3, pp. 487-93, Mar 2007.

[50] R. M. Kessler, J. R. Ellis, Jr. and M. Eden, "Analysis of emission tomographic scan data: limitations imposed by resolution and background," J Comput Assist Tomogr, vol. 8, no. 3, pp. 514-22, Jun 1984.

[51] N. Li and L. J. Meng, "Adaptive Angular Sampling for SPECT Imaging," IEEE Trans Nucl Sci, vol. 58, no. 5, pp. 2205-2218, Oct 2011.

[52] B. Auer, N. Zeraatkar, J. C. Goding, A. Könik, T. J. Fromme, K. S. Kalluri, L. R. Furenlid, P. H. Kuo and M. A. King, "Inclusion of Quasi-Vertex Views in a Dedicated Brain-SPECT System for Improved Imaging Performance," Phys Med Biol, Under Review, 2020.

[53] M. C. Goorden, M. C. Rentmeester and F. J. Beekman, "Theoretical analysis of full-ring multi-pinhole brain SPECT," Phys Med Biol, vol. 54, no. 21, pp. 6593-610, Nov 072009.

[54] P. Nillius and M. Danielsson, "Theoretical bounds and system design for multipinhole SPECT," IEEE Trans Med Imaging, vol. 29, no. 7, pp. 1390-400, Jul 2010

[55] N. Zeraatkar, L. Furenlid, G. Zubal and M. King, "Temporal shuttering of apertures to enhance axial and angular sampling in a multi-pinhole SPECT system for brain imaging," Journal of Nuclear Medicine, vol 59, no. supplemenet 1, p. 585, 2018.

[56] N. Zeraatkar, B. Auer, K. Kalluri, N. Momsen, L. Furenlid, P. Kuo et al., "Effect of truncated overlapping projection data in a multi-pinhole brain SPECT system with temporal shuttering of apertures," Journal of Nuclear Medicine, vol. 60, no. supplement 1, pp. 39-39, 2019.
[57] K. S. Kalluri, N. Zeraatkar, B. Auer, P. H. Kuo, L. R. Furenlid and M. A. King, "Preliminary investigation of AdaptiSPECT-C designs with square or square and hexagonal detectors employing direct and oblique apertures", Proc. SPIE 11072, 15th International Meeting on Fully Three-Dimensional Image Reconstruction in Radiology and Nuclear Medicine, 1107215 (28 May 2019). 